Document downloaded from:

http://hdl.handle.net/10251/142895

This paper must be cited as:

Sharma, S.; Broatch, A.; Garcia Tiscar, J.; Allport, JM.; Nickson, AK. (12-2). Acoustic characterisation of a small high-speed centrifugal compressor with casing treatment: an experimental study. Aerospace Science and Technology. 95:1-15. https://doi.org/10.1016/j.ast.2019.105518

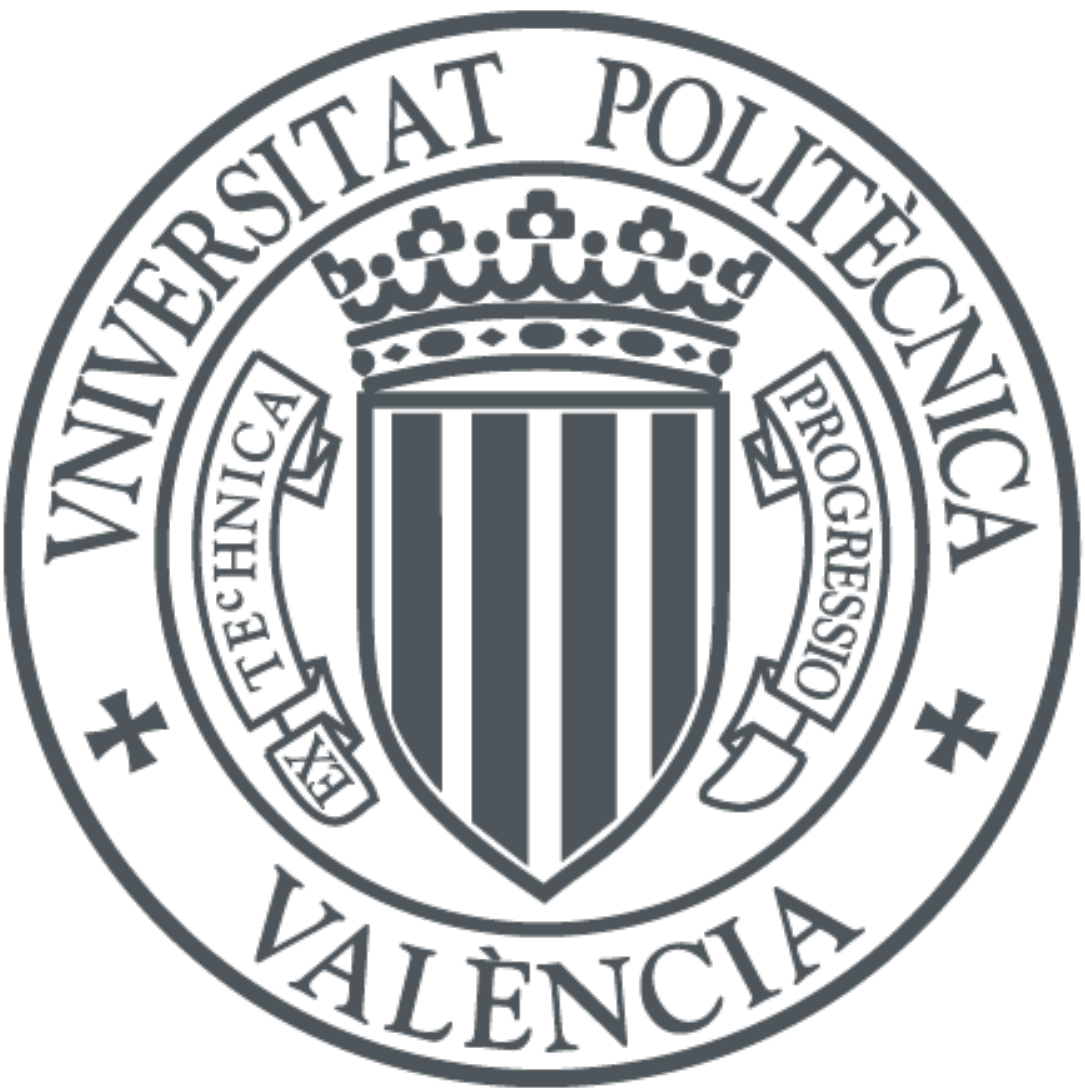

The final publication is available at

https://doi.org/10.1016/j.ast.2019.105518

Copyright Elsevier

Additional Information 


\title{
Acoustic characterisation of a small high-speed centrifugal compressor with casing treatment: An experimental study
}

\author{
S. Sharma ${ }^{\mathrm{a}, *}$, A. Broatch ${ }^{\mathrm{b}}$, J. García-Tíscar ${ }^{\mathrm{b}}$, J. M. Allport ${ }^{\mathrm{a}}$, A. K. Nickson ${ }^{\mathrm{c}}$ \\ ${ }^{a}$ Turbocharger Research Institute, University of Huddersfield, Queensgate, Huddersfield HDI 3DH, United Kingdom \\ ${ }^{b}$ CMT - Motores Térmicos, Universitat Politècnica de València, Camino de Vera, 46022 Valencia, Spain \\ ${ }^{c}$ Borg Warner Turbo Systems Division, Bradford, West Yorkshire BD4 6SE, United Kingdom
}

\begin{abstract}
With the advancements in manufacturing, materials and computational methods, turbomachinery designs have become more efficient providing higher specific power and reliability with lower weight and cost. The rotational speed of turbomachines has increased while the overall machine size has decreased for a similar power output leading to the pervasive presence of small, high-speed turbomachines, specifically centrifugal compressors in helicopters, unmanned aerial vehicles (UAVs), auxiliary power units (APUs), turbochargers and micro gas turbines. In addition to superior aerodynamic performance over a wide range, increased operating speeds have made the acoustic emissions of small centrifugal compressors a critical aspect of design. Therefore, this work presents an experimental campaign to characterise the acoustic behaviour of a compressor with an intent to quantify the dominant features of the flow-induced noise for design and near surge operating conditions. Furthermore, the campaign is extended to establish the impact of the ported shroud casing treatment and operating speed on the acoustic emission of the compressor. The in-duct noise measurement method is used in this work to quantify the noise generated in the compressor by measuring pressure fluctuations near the inducer and diffuser while the propagation of the generated noise to the ducts is computed from an array of piezoelectric sensors. Spectra at the design operating point are dominated by tonal noise while broadband noise content is the dominant feature of spectra for near surge operation. Although the ported shroud cavity does not significantly alter the overall noise levels of the compressor operating at design condition, it does seem to propagate tonal noise. For near surge operation, the casing treatment positively impacts the acoustic emission with a reduction of approximately $10 \mathrm{~dB}$ in the range up to the blade pass frequency. Furthermore, various broadband features are also observed to be alleviated by the casing treatment.
\end{abstract}

Keywords: Aeroacoustics, Noise measurement, Compressor, Surge, Ported Shroud

\section{Introduction}

Turbomachines have a surprisingly long association with the technological growth seen in human industrial society. The specific power output of these turbomachines has increased over time and small high-speed turbomachines

\footnotetext{
*Corresponding author. Tel.: +44 148447 1084, email: sidharath.sharma@hud.ac.uk 


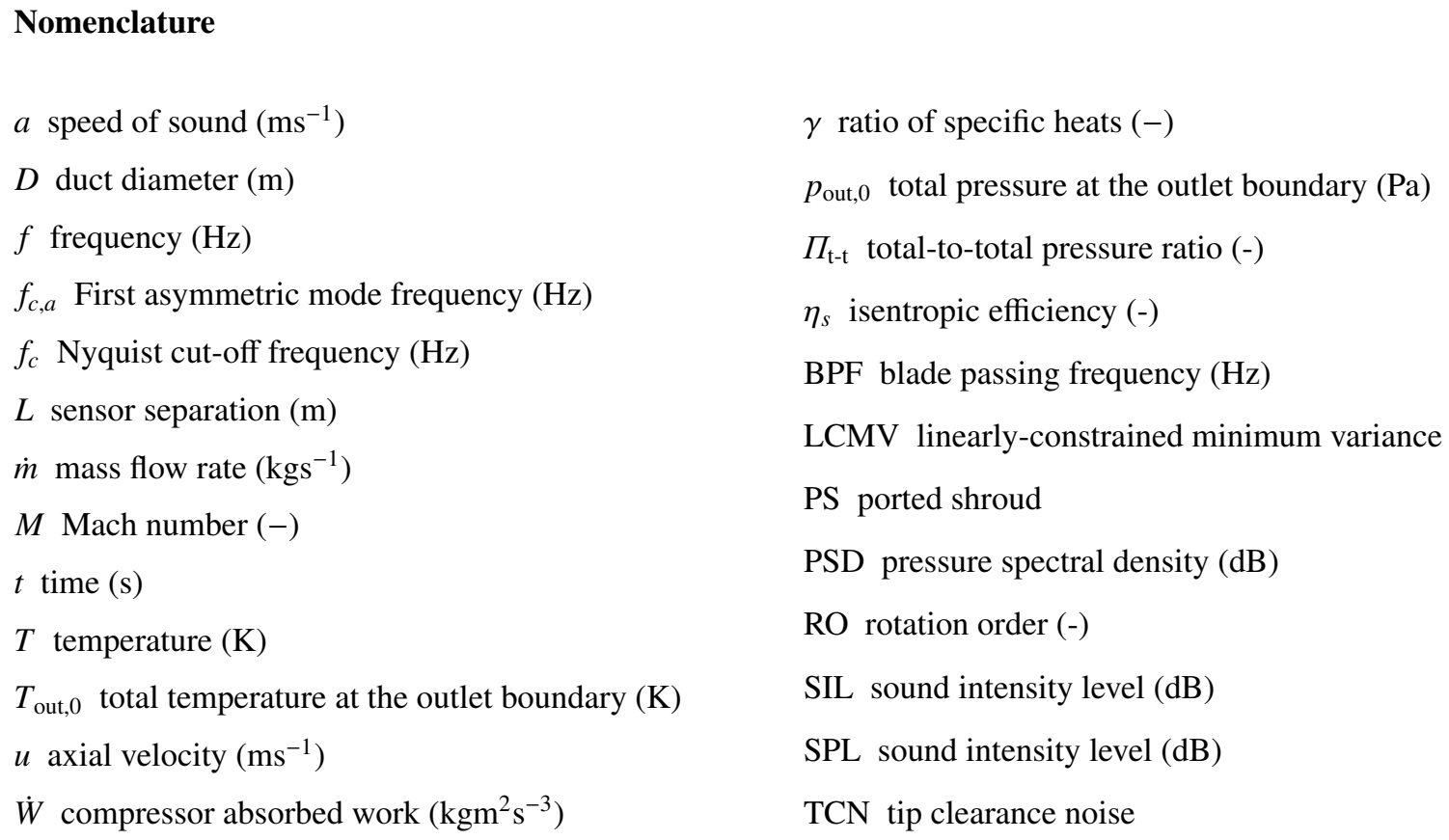

$\gamma$ ratio of specific heats $(-)$

$p_{\text {out }, 0}$ total pressure at the outlet boundary $(\mathrm{Pa})$

$\Pi_{\mathrm{t}-\mathrm{t}}$ total-to-total pressure ratio (-)

$\eta_{s}$ isentropic efficiency (-)

BPF blade passing frequency $(\mathrm{Hz})$

LCMV linearly-constrained minimum variance

PS ported shroud

PSD pressure spectral density $(\mathrm{dB})$

RO rotation order (-)

SIL sound intensity level (dB)

SPL sound intensity level (dB)

TCN tip clearance noise

are increasingly developed owing to the incorporation of innovative control technologies, specialised materials and sophisticated aerodynamic designs. Centrifugal machines, specifically compressors, are better suited for smaller sizes and hence, small high-speed turbomachines are dominated by centrifugal configurations. One can find these machines in a range of applications that include helicopters, unmanned aerial vehicles, micro gas turbines, turbochargers, vacuum cleaners, hair dryers and even artificial heart pumps.

As the change in sound power is proportional to the fifth or sixth power of the change in rotor tip speed, the acoustic emission of these small high-speed centrifugal compressor is critical to the success of the product. These machines are expected to deliver good aerodynamic performance over a wide range of operation with lower acoustic emissions. The most widely used of these small turbomachines with extensive development is the ported shroud (PS) automotive turbocharger compressor. Therefore, noise generated in such a compressor is investigated in this work.

Ported shroud self-recirculating casing treatment is a widely used passive flow control method to delay the onset of surge. The increase in the stable operating region of the compressor is achieved by recirculating the low momentum fluid that blocks the blade passage to the compressor inlet through the ported shroud cavity [1, 2, 3]. Although the use of a ported shroud enhances the aerodynamic stability, its impact on the acoustic characteristics is an area of active research.

The literature on the acoustic characterisation of the compressors can be primarily divided into internal or induct noise measurements and external or radiated noise measurements. The internal noise is measured by dynamic pressure sensors in direct contact with the internal flow by placing them inside the compressor and/or ducts while 
external noise measurements capture the noise radiated by the compressor using microphones positioned in the far field and/or near field or duct orifice.

Another differentiation can be made on the parameter used to quantify the noise emitted by the system viz. Sound Pressure Level (SPL) and Sound Intensity Level (SIL). While the former is a scalar term representing the sum total of noise at a particular location, the latter is a vector quantity whose directivity properties can be used to capture the noise emitted from a particular direction. Sound pressure levels can be measured with a single sensor and are susceptible to issues caused by reflections and the mode shapes of the geometry. The determination of sound intensity levels necessitates two or more sensors and remains consistent along the geometry due to strong directivity properties.

External noise measurements were performed by Evans and Ward [4], Sun et al. [5, 6], Teng and Homco [7], Sun et al. [8], Lee et al. [9], and Pai et al. [10, 11] using free field microphones to measure the SPL of either the radiated far field noise or duct orifice noise emitted by a turbocharger compressor. Internal or in-duct noise measurements were seen in the works of Gaudé et al. [12], Tiikoja et al. [13], Raitor and Neise [14], Broatch et al. [15], Torregrosa et al. [16] and Sun et al. [8]. Trochon [17] and Evans and Ward [4] also presented in-duct noise plots, implying a coherence between internal and external noise measurements. Tiikoja et al. [13] used an acoustic two port method to determine the SPL of the noise generated by the compressor while Raitor and Neise [14], Trochon [17] and Evans and Ward [4] measured SPLs using a single sensor.

Gaudé et al. [12] used arrays of pressure sensors for the inlet and outlet ducts in order to decompose the in-duct pressure wave to determine SIL. A similar approach was then used by Broatch et al. [15, 18] and Torregrosa et al. $[16,19]$ demonstrating the robustness of a beamforming based pressure decomposition approach over conventional two port and single sensor methods. The authors $[16,19]$ also demonstrated a good correlation between internal and external noise measurement results.

Although the external noise measurements are relatively straightforward to set up due to their non-intrusive nature, they do require anechoic conditions to yield accurate results. On the contrary, internal noise measurements are relatively challenging to set up due to their intrusive nature but are free from the necessity of anechoic conditions. Additionally, the external radiated noise is not solely dependent on the noise generated by the flow but is also influenced by the geometry and the radiative properties of the material. An understanding of the flow mechanisms responsible for noise is the larger objective of this work and, therefore, internal or in-duct noise measurements are preferred to establish the acoustic characteristics of the compressor.

Specific tonal and broadband acoustic features are consistently identified throughout the experimental literature on the noise spectra of compressors. The tonal features include Rotating Order (RO) ${ }^{1}$ or 'buzz-saw' tones [14] and Blade Pass Frequency $(\mathrm{BPF})^{2}$ while broadband noises are identified as the onomatopoeic 'whoosh' noise [4, 7, 20] observed within the plane wave range and Tip Clearance Noise (TCN) [13] present outside the plane wave range. Some authors $[21,22]$ seem to conflate these two broadband features into a single inclusive broadband but they appear to be two

\footnotetext{
${ }^{1}$ Also known as rotational frequency, $\mathrm{RO}=n / 60$ where $n=$ rotational speed of the impeller in rotations per minute (rpm)

${ }^{2} \mathrm{BPF}=\mathrm{RO} \times$ number of impeller main blades
} 
different phenomena. The lower frequency 'whoosh' noise can be identified within $1-4 \mathrm{kHz}$ range while TCN is usually seen at higher frequencies before the BPF of the compressor.

The literature on the acoustic impact of casing treatment is scarce, and the general perception [23, 24] is that the casing treatment deteriorates the acoustic spectra of the compressor by increasing the overall noise emission. Chen and Yin $[23,24]$ pointed out the 'noise issue', specifically BPF as the reason for the limited use of casing treatment in the compressors used in the turbochargers of passenger cars without providing any evidence for these claims.

Recent studies $[25,26]$ on the acoustic impact of the casing treatment were published with somewhat contrary results. Dehner et al. [25] experimentally investigated the impact of ported shroud casing treatment and observed a decrease in the overall noise levels at the inlet duct for design operation despite increased tonal content by using casing treatment. For near surge or low flow conditions, an increase in the overall noise levels was seen. The spectra measured at the outlet duct for the ported shroud compressor showed little difference at near surge conditions while a decrease was again seen at design conditions. It is worth pointing out that the casing treatment used in this work decreased the stable flow region of the compressor rather than increasing it. Fardafshar and Koutsovasilis [26] explored the impact of the ported shroud by modelling the near-field spectra of the compressors with and without casing treatment at near surge and choke condition. The results showed higher noise levels for the compressor without casing treatment for near surge operation while lower noise levels for the same compressor were seen for choke operation.

\section{Methodology}

An internal or in-duct noise measurement method similar to the one presented by Broatch et al. [15] was employed to characterise the acoustic behaviour of the compressor. Both single sensor and multi-sensor methods were considered in this work for exploring noise generation and noise propagation respectively. The noise generated in the compressor was measured at the impeller upstream and downstream positions by miniature pressure probes. The noise propagated at the compressor ducts was quantified from pressure spectra obtained from the sensor arrays consisting of three piezoelectric sensors each for inlet and outlet ducts. The pressures measured by these arrays were further processed to decompose pressure waves into their corresponding components by the beamforming based approach.

The sound intensity measurements were based on the assumption that the pressure signal $p(t)$ recorded at a point in a duct is a linear superposition of two opposite waves travelling across each other with a phase difference of $180^{\circ}$. These wave components $p^{+}(t)$ and $p^{-}(t)$ are commonly referred to as forward and backward wave components respectively.

$$
p(t)=p^{+}(t)+p^{-}(t)
$$

The sound intensity at that point in the duct was then calculated with the help of the expression proposed by 
Morfey [27]:

$$
I=\frac{1}{\rho a}\left[\left|P^{+}\right|^{2}\left(1+M^{2}\right)-\left|P^{-}\right|^{2}\left(1-M^{2}\right)\right]
$$

where $P^{+}$and $P^{-}$are the respective complex frequency spectra of $p^{+}(t)$ and $p^{-}(t)$ wave components, $\rho$ is the mean density, $a$ is the mean speed of the sound and $M$ is the mean Mach number of the flow.

In order to compute the sound intensity, the measured pressure wave had to be decomposed into its forward and backward travelling components. This was achieved with an understanding of the wave propagation through the duct in each direction, gained by comparing the pressure data for two or more subsequent spatial positions. One such wave decomposition method is beamforming.

\subsection{Beamforming wave decomposition}

The beamforming based wave decomposition provides a mathematical method to 'steer' the content of a pressure signal into the forward and backward component. This decomposition method is based on the idea that the direction in which a particular wave originates impacts the time when it would be recorded by each sensor of a linear array. This is demonstrated in Fig. 1, where a reference array encountering a plane wavefront originated in the direction closer to sensor 1 is shown to travel an extra distance to be measured by sensor 2 and sensor 3 . This extra distance would lead to time and phase delay. Therefore, by comparing the simultaneous waves recorded by these sensors, the origin of the wavefront can be computed. Among the available beamformers, the Delay-Sum and Linearly Constrained Minimum Variance (LCMV) methods were explored, and the narrowband LCMV beamformer was selected for this work. The implementation of LCMV beamformer is based on the work of Broatch et al. [15] and Torregrosa et al. [19]. The details of the mathematical procedure are available from Torregrosa et al. [19] and García-Tíscar [28].

For effective implementation of these beamforming arrays, a few practical concerns must be considered. Firstly, beamforming is based on the assumption that the decomposed wave is propagating within the plane wave region. Therefore, sufficient straight length should be provided before and after the sensor array to ensure plane wave flow at the arrays. Torregrosa et al. [29] based on their experience of applying beamforming on multiple problems, recommended a minimum of 6 and 4 diameters straight length upstream and downstream of the array respectively. Another consideration regarding the position of array's sensor is to avoid the $1 / 5$ and $1 / 3$ nodes of the standing wave pattern in the duct.

One-dimensional plane wave propagation of the flow is limited by the onset of acoustic modes that introduces three-dimensional effects in the flow propagation. The limiting frequency for the beamforming and thereby, sound intensity can be predicted by computing the onset of these acoustic modes from the formulations provided by Eriksson [30]. According to Eriksson [30], the wave propagation in a circular duct can be assumed of a planar or one dimensional nature below a particular cut-off frequency which marks the onset of first asymmetric mode defined as

$$
f_{c, a}=1.84 \frac{a}{\pi D} \sqrt{1-M^{2}}
$$




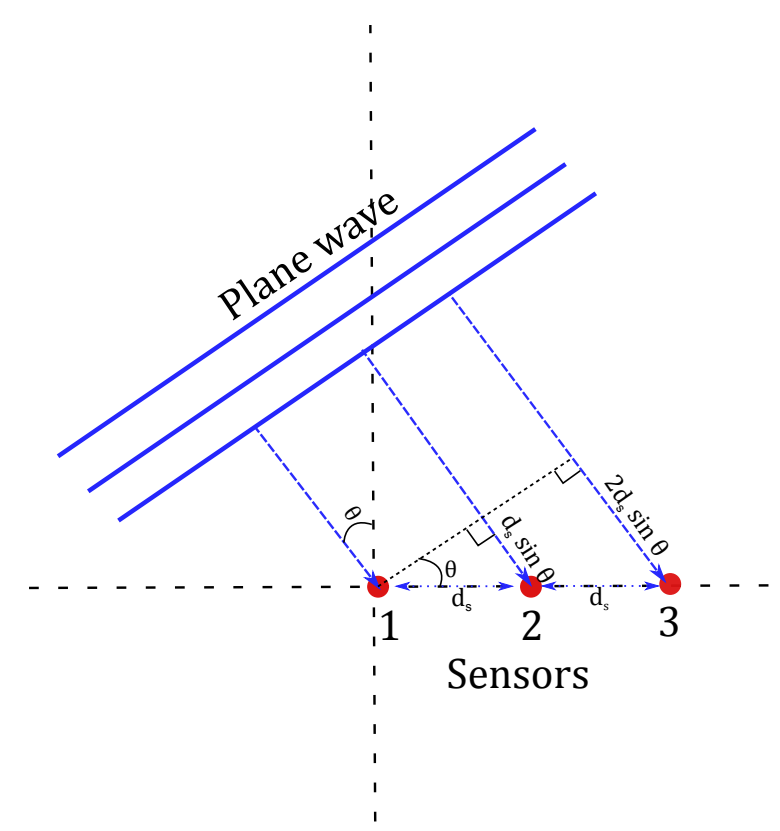

Figure 1: Propagation of a planer wavefront moving with a velocity ' $a$ ' towards an array of receivers. If $d_{s}$ is the distance between two successive sensors, a delay corresponding to the distance of $d_{s} \sin \theta$ would be introduced.

where $D$ is the duct diameter, $a$ is the speed of sound and $M$ is the mean Mach number of the flow. The next acoustic mode is the radial mode, and its propagation is computed by

$$
f_{c, r}=3.83 \frac{a}{\pi D} \sqrt{1-M^{2}}
$$

Other than the onset of the higher order modes discussed above, beamforming is also limited by a Nyquist like frequency criterion dictated by the spatial resolution of the sensor array. Spatial aliasing effects are seen above this Nyquist cut-off frequency, which is defined as less than half of the inherent frequency of the array [31]. As seen in Fig. 1, the time delay between two subsequent sensors with spacing $d_{s}$ is $d_{s} \sin \theta / a$. The inherent frequency for a perpendicular wave front can then be computed as $a / d_{s}$. Therefore, the cut-off for Nyquist like frequency criterion can be defined as

$$
f_{c, N}<\frac{f_{\text {array }}}{2}=\frac{a}{2 d_{s}}
$$

Furthermore, the spacing $d_{s}$ between sensors in the array is a compromise between high frequency and low frequency characteristics of the array. If $d_{s}$ is decreased to improve the Nyquist limitations, the low frequency spatial resolution would be deteriorated as the wavelength at lower frequencies could exceed the distance between the sensors; defying the whole purpose of the array. Finally, the sensors should be flush mounted on the straight ducts and properly calibrated to give a coherent response. 


\section{Measurement set-up}

The series $B 2 G$ turbocharger unit and digital geometry of the corresponding compressor were provided by industrial partner BorgWarner [32]. The compressor of this particular model incorporates Ported Shroud (PS) casing treatment with three symmetric structural struts supporting the cavity. The front and section views of the compressor geometry are shown in Fig. 2. The impeller features seven main and seven splitter blades that are designed as forward swept disseminating the flow into a vaneless diffuser.
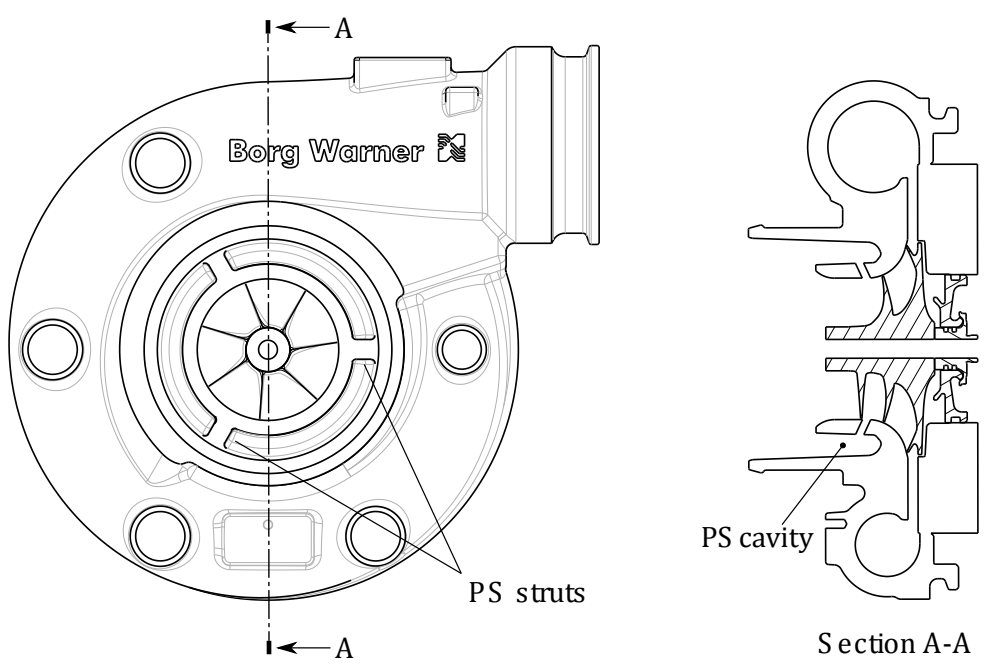

Figure 2: Drawing of the investigated ported shroud compressor, showing the location of the supporting struts and the cavity geometry.

To quantify the impact of the PS on the acoustic emission of the compressor, the inlet of the original or baseline compressor shown in Fig. 2 is altered to block the PS slot. The state of the compressor with PS slot similar to the original configuration is referred as PS open configuration while the configuration with modified PS slot is called as PS blocked configuration. These configurations shown in Fig. 3 are realised by modifying the original compressor with a modular inlet design which uses two inlet inserts, one with the PS slot and one without PS slot for PS open and blocked configuration respectively. The modular design makes it feasible to retain compressor components other than the inlet insert for the two configurations; thereby, providing minimal variability in the experiments due to the manufacturing tolerances of these components.

The performances of the aforementioned PS open and blocked compressor configurations were characterised by measuring the thermodynamic variables of the state attained by fixing the air mass flow rate and the rotational speed of the impeller. The performance measurements were performed at the gas stand testing facility in BorgWarner Turbo Systems, Bradford. Pressure and temperature values were recorded for multiple operating points achieved through incremental reduction of air flow whilst maintaining a fixed rotational speed. The air mass flow rate was regulated by the operation of a back pressure valve at the compressor outlet while the rotational speed of the compressor was regulated from the turbine end by controlling the conditions at the inlet of the turbine. Further details on the gas stand 


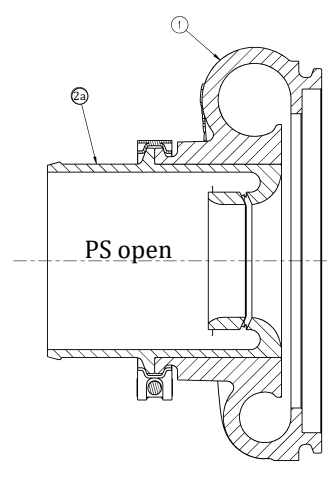

1 - Compressor cover

2a-PS open Insert

$2 \mathrm{~b}$-PS blocked Insert

3 -V-clamp
4 -Nut
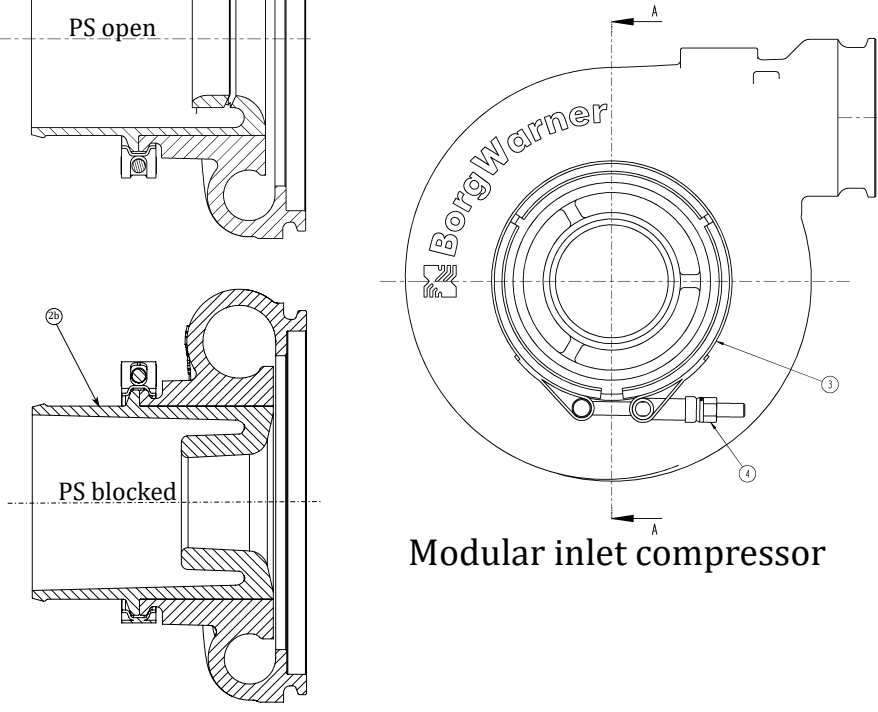

Modular inlet compressor

Figure 3: Drawing of the compressor fitted with the modular inlet. The section views of the compressor with inlet inserts corresponding to PS open and blocked configurations are also shown.

measurements are described in Sharma et al. [33]. Following this procedure, the thermodynamic state values were recorded for various rotating speeds ranging from $55 \mathrm{krpm}$ to $130 \mathrm{krpm}$.

The measured air mass flow and compressor rotational speed values were corrected for Standard day conditions to account for the variations in ambient conditions on the day of measurements. The corrected mass flow rate and rotational speed values were computed using the following formulation:

$$
\begin{aligned}
\dot{m}^{*} & =\dot{m}\left[\frac{p_{r e f}}{p_{i n, 0}}\right] \sqrt{\frac{T_{i n, 0}}{T_{r e f}}} \\
N^{*} & =N \sqrt{\frac{T_{r e f}}{T_{i n, 0}}}
\end{aligned}
$$

where $N$ is compressor rotating speed, $\dot{m}$ is the air mass flow rate, $T_{i n, 0}$ is the total temperature at the compressor inlet, $p_{i n, 0}$ is total pressure at compressor inlet, $T_{r e f}$ is $288.15 \mathrm{~K}$ and $p_{r e f}$ is $1 \mathrm{~atm}$.

The measured thermodynamic variables of each state are then used to compute compressor performance parame- 


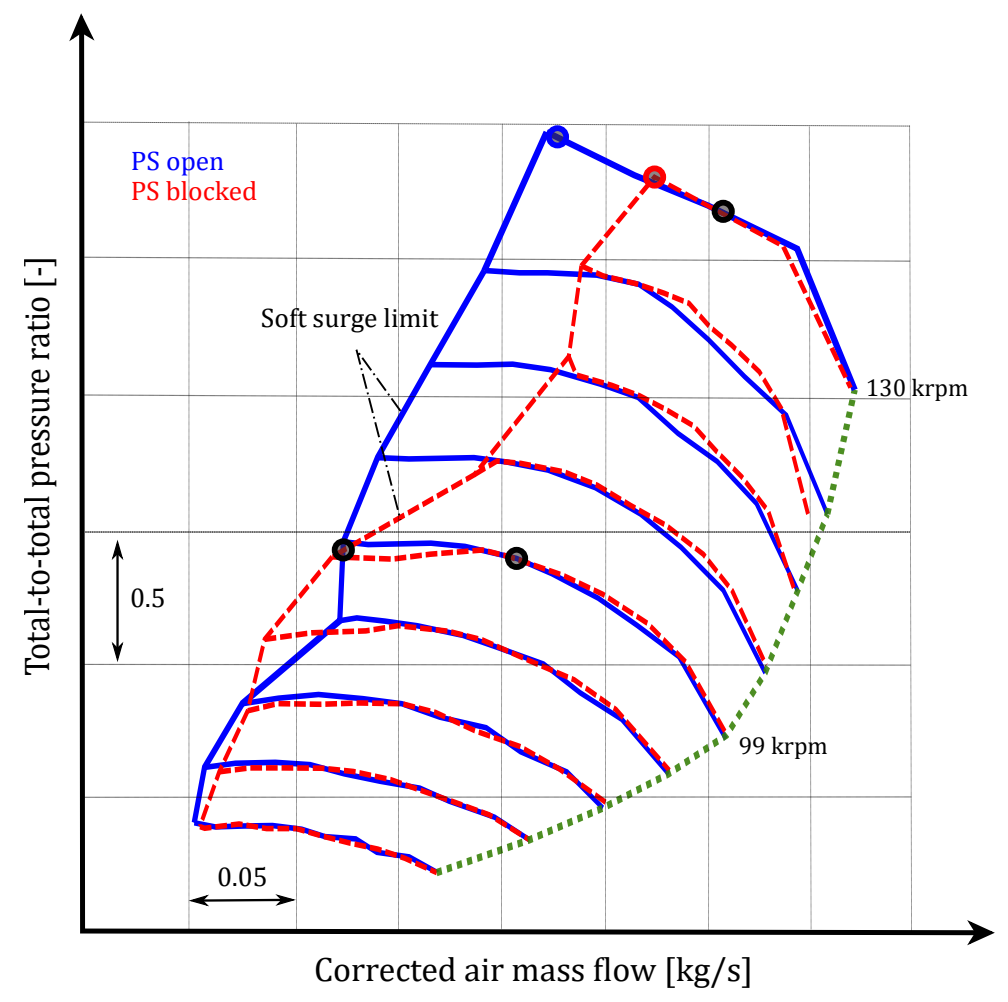

Figure 4: Experimental compressor maps for the open and blocked configurations, highlighting the investigated operating points which include design and near surge conditions of $99 \mathrm{krpm}$ and $130 \mathrm{krpm}$ speedlines in both configurations.

ters viz. total-to-total pressure ratio $\Pi_{t-t}$ and isentropic efficiency $\eta_{s}$.

$$
\begin{aligned}
\Pi_{t-t} & =\frac{p_{\text {out }, 0}}{p_{\text {in }, 0}} \\
\eta_{s}=\frac{\dot{W}_{i s}}{\dot{W}} & =\frac{T_{\text {in }, 0}}{T_{\text {out }, 0}-T_{\text {in }, 0}}\left(\Pi_{t-t}^{\gamma-1 / \gamma}-1\right)
\end{aligned}
$$

Total to total pressure ratio against the corrected air mass flow rate for the two compressor configurations are shown in Fig. 4, commonly known as compressor maps. It is worth mentioning that the surge limit identified in this map is not that of deep surge but rather mild or soft surge [34] which is characterised by low-amplitude flow oscillations in the system along with slight decline in the isentropic efficiency. This limit is established by adding a safety margin to the deep surge point ascertained by pressure spike and is provided as a safety reference for engine manufacturers and integrators. The extension of the surge limit can be clearly seen in the PS open configuration, especially at higher operating speeds.

The impact of PS on the acoustic emission of the compressor operating at design conditions can be established rather simply, by comparing the acoustic spectra of PS open and blocked configuration as the design points for two configurations are aerodynamically similar. On the contrary, surge limits and thereby, corresponding near surge points for two configurations are very different, and therefore, a direct comparison between the acoustic spectra of the two 
configurations operating near surge would not be an accurate means to quantify the impact of PS. The differences in the acoustic spectra of the near surge points for two configurations would not be limited to the deviations in the PS geometry but an amalgamation of the alterations caused by differences in geometries and aerodynamic phenomena due to different mass flow rates and corresponding thermodynamic variables. One way around this problem is the determination of two acoustically similar near surge states that can be meaningfully compared to establish the impact of the PS on the acoustic emission of near surge operation. The comparable near surge operating points can be determined by applying acoustic similarity laws [35, 36, 37, 38]. Acoustic similarity laws are not used in this work as coincidently, near surge points of $99 \mathrm{krpm}$ speed lines for two configurations are similar as seen in the compressor map.

Therefore, design and near surge operation for PS open and blocked configurations at $99 \mathrm{krpm}$ are investigated to quantify the impact of the PS on the acoustic emission of the compressor. Furthermore, the compressor operation at $130 \mathrm{krpm}$ is also studied to understand the evolution of acoustic spectra with an increase in rotational speed. The 99 $\mathrm{krpm}$ and $130 \mathrm{krpm}$ iso-speedlines are also referred to as low and high speedline respectively in this work.

\subsection{Acoustic flow bench}

The acoustic measurements presented in this work were carried out in a flow bench facility hosted in CMT Motores Térmicos. The flow bench uses a $150 \mathrm{~kW}$ screw compressor which was powered by a 10L Diesel engine. The compressed air was first passed through an charge air cooler and then collected in a reservoir to ensure a steady supply. The collected air in the reservoir was then routed to the engine and the turbine of the turbocharger. Therefore, the screw compressor acts as a supercharger for the Diesel engine as well as provides the air flow to the turbine of the installed turbocharger. The combination of the mass flow rate at the turbine inlet and back pressure valve located at the compressor outlet was used to fix the operating point of the compressor unit of an installed turbocharger. The thesis of García-Tíscar [28] can be referred for further details on this flow bench.

For the measurements, the turbocharger with appropriate compressor configuration was installed on the flow bench. The inlet and outlet of the compressor were fitted with long straight pipes to ensure sufficient development of flow suitable for a plane wave to occur at the measurement sections. The diameters of the ducts were appropriate to preserve a cross-sectional area of the adjacent compressor inlet/outlet section to avoid complex three-dimensional structures caused by singularities and impedance mismatch. Two piezoelectric sensor arrays consisting of three piezoelectric sensors each were positioned, one each at the inlet and outlet duct measurement section. The average pressure and temperature values were measured at the compressor inlet and outlet sections while the air mass flow rate was only measured at the compressor inlet. A schematic of the compressor measurement layout showing various sensors and their positions are shown in the Fig. 5.

In addition to the sensors positioned in inlet and outlet ducts, the compressor was also instrumented to measure the rotational speed and to quantify the noise generated by the impeller. For the purpose of the noise generation, two miniaturised pressure probes were positioned in the inducer and diffuser of the compressor. The inducer probe was 


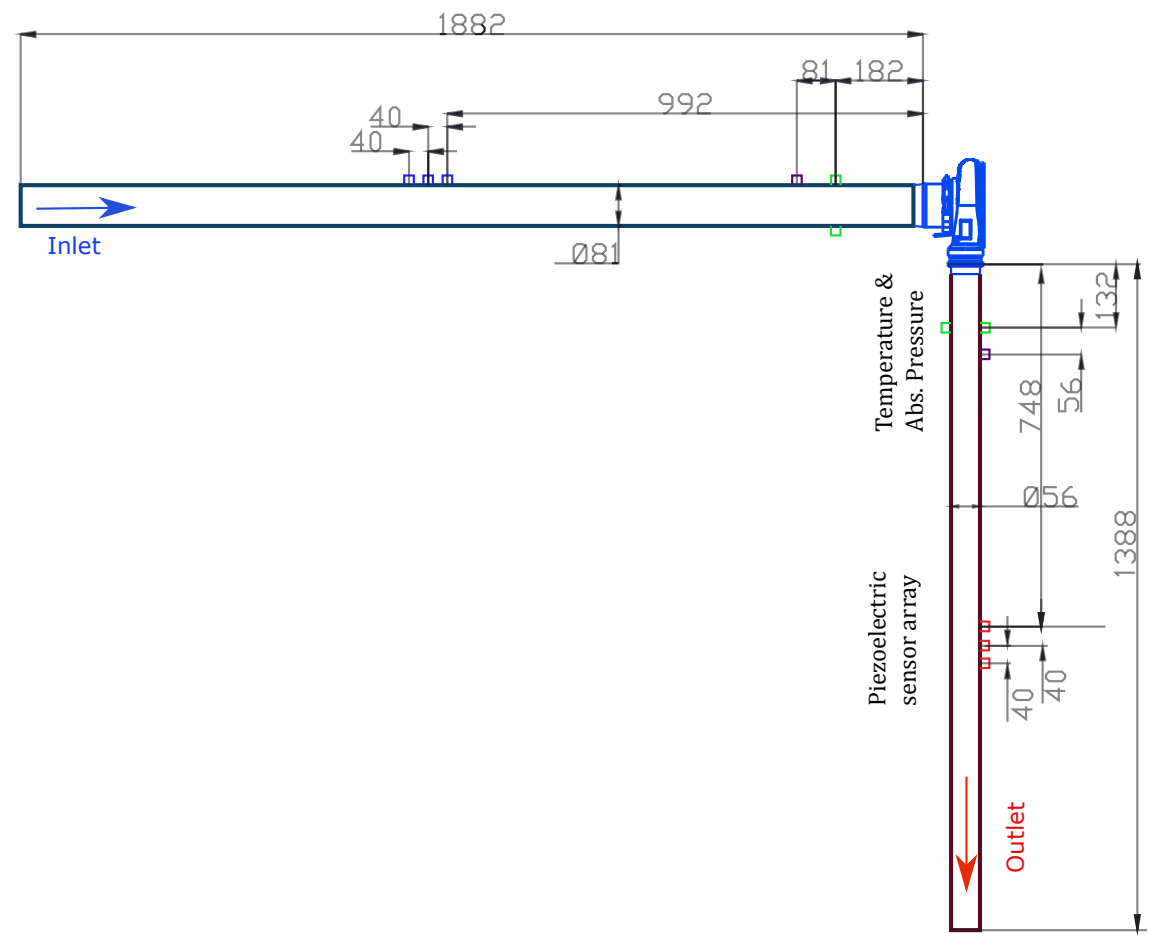

Figure 5: Schematic of the test rig used for measuring the acoustic characteristics of the compressor is shown. The dimensions marked are in millimetres $(\mathrm{mm})$. The location of various pressure and temperature instrumented on the rig are also shown.

wall flushed in the PS cavity, and the diffuser probe was wall flushed at the middle along the length of the diffuser. The instrumented compressor along with the position of various probes are shown in Fig. 6.

Dynamic pressure fluctuations obtained from the piezoelectric sensor arrays and miniaturised pressure probes were recorded for 1s with a sampling frequency of $200 \mathrm{kHz}$ using a Yokogawa DL850V recorder. The global variables of the experimental set-up were measured with an in-house custom-built data acquisition system that averages the sensor data over 5s. These parameters include air mass flow, turbo speed, static temperatures and absolute static pressures of the air flow with the last two variables being recorded for both inlet and outlet ducts. Temperature data is an average of thermocouples placed at different radial locations of the same cross-section.

Measured dynamic pressure fluctuations were processed to obtain the acoustic characteristics of the compressor. The pressure spectra represented in terms of Power Spectral Density (PSD) were obtained using Welch's overlapped segmented averaging [39] of the pressure data. The number of blocks were selected with an intent to achieve a frequency resolution of approximately $50 \mathrm{~Hz}$. These blocks were then tapered using Hanning functions with $50 \%$ overlap. Spectra of the decomposed pressure wave components were computed similarly to calculate the PSD of the sound intensity. 


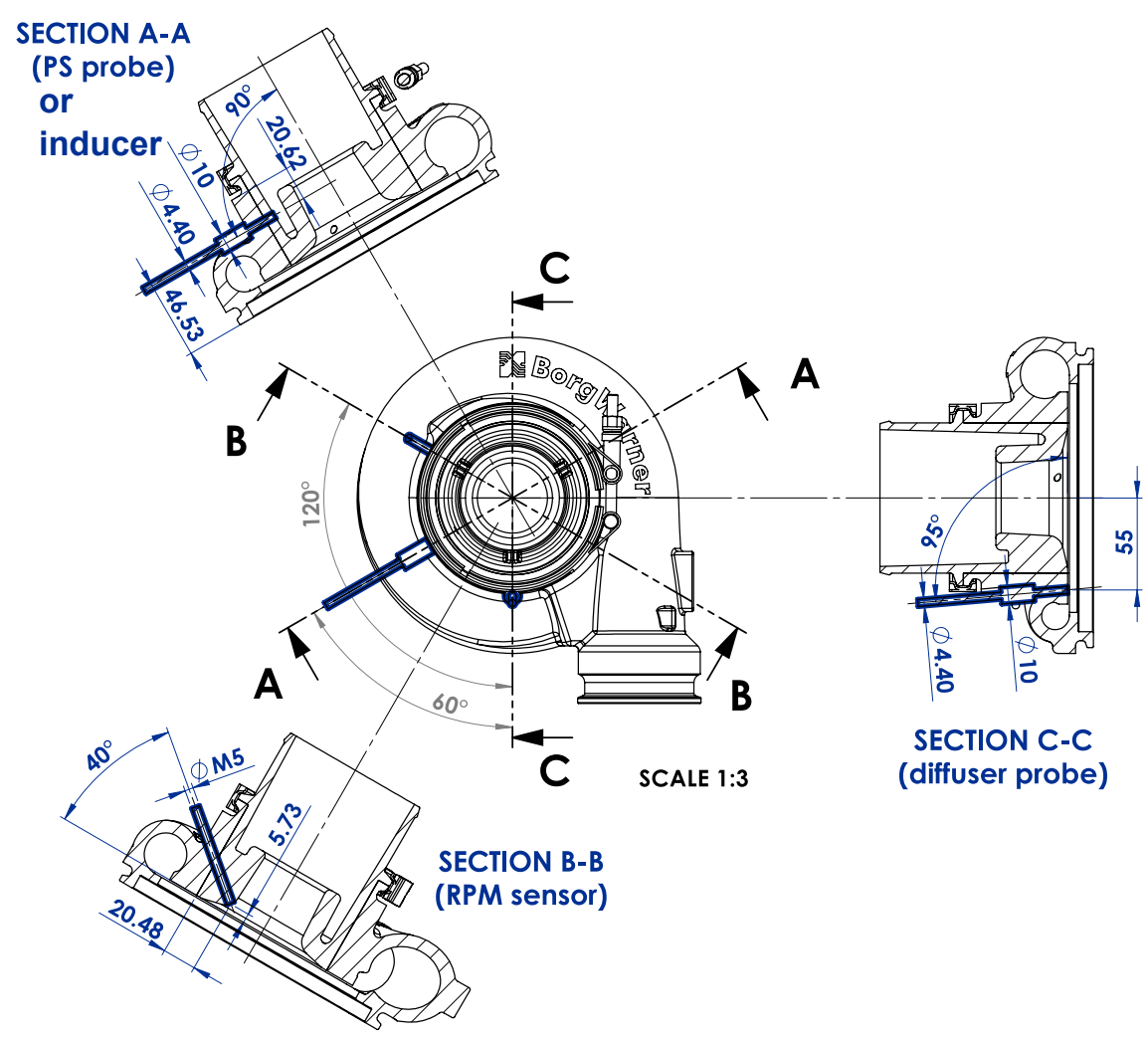

Figure 6: Instrumentation on the turbocharger compressor, highlighting three sections corresponding to the position of inducer probe inside PS cavity, diffuser probe and RPM sensor.

\section{Results and discussion}

Acoustic characteristics of open and blocked PS configurations of the compressor operating at the design and near surge conditions for two aforementioned iso-speed lines viz. $99 \mathrm{krpm}$ and $130 \mathrm{krpm}$ are analysed in this section.

It is worth pointing out the spectra computed from standalone experimental pressure signals and the corresponding beamformed experimental signal are observed to be well correlated as shown in Fig. 7 implying well developed flow at the measurement sections of the ducts. Moreover, due to plane wave restriction for the beamforming, spectra from the each sensor of the duct array is utilised in this work.

\subsection{9 krpm speedline}

The acoustic characteristics of the PS open and blocked compressor configurations operating at the Design or Best Efficiency Point (BEP) and Near Surge (NS) conditions for $99 \mathrm{krpm}$ iso-speedline are presented here. As discussed before, the operating points of this speedline are aerodynamically similar for both configurations and therefore, the results can be directly compared. 

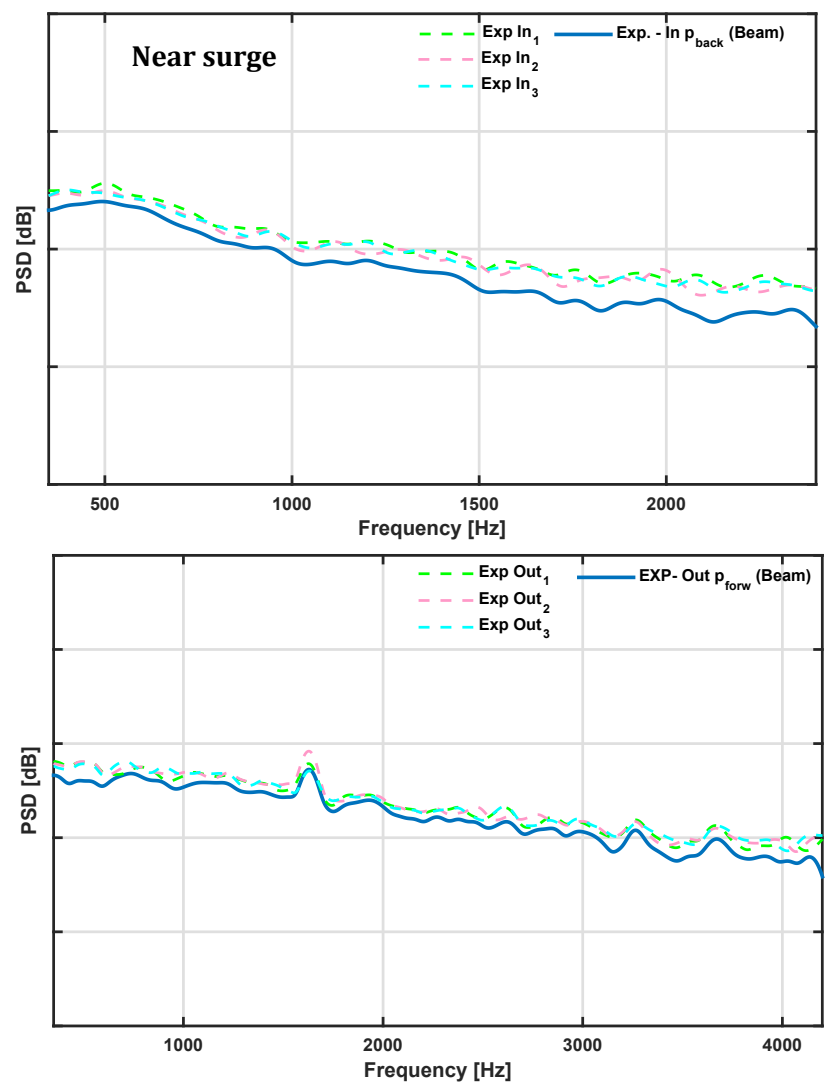

Figure 7: Comparison of the inlet (top) and outlet (bottom) duct spectra up till plane wave limit, obtained from the individual sensors and beamforming for near surge condition of lower speedline for PS open configuration. Y-axis scale is maintained at $20 \mathrm{~dB}$ per division.

\subsubsection{PS open configuration}

Pressure spectra computed from piezoelectric arrays and miniature pressure probes for PS open compressor operating at the design and near surge conditions are presented in Fig. 8 and Fig. 9 respectively. The noise generated by the impeller is investigated from the spectra of inducer and diffuser probe while the propagation of the noise in to the compressor ducts is analysed from the spectra of duct arrays.

Design operation. The pressure spectra of the probes located at the upstream of the impeller for the compressor operating at design condition (top of Fig. 8) shows that the noise generated by the PS open configuration operating at the design condition as seen from the inducer spectrum is relatively flat with multiple tonal features that include the harmonics of RO and BPF. In addition to expected RO tones, a low-frequency peak at approximately $0.4 \mathrm{RO}$ is also seen in the inducer spectrum.

The inlet spectra reasonably follow the inducer spectrum with an expected decrease in the levels. Broadband elevation in the characteristic 'whoosh' noise frequency range is not observed in either inducer or inlet duct spectra. The spectra of the individual sensors of the inlet piezoelectric array show good correlation, specifically between the spectra of the second and third probe. The spectrum of the first inlet probe deviates from the other two in terms of 
amplitude from $8.2 \mathrm{kHz}$. Broadband elevation in the range of $13.7-15.7 \mathrm{kHz}$ (highlighted as 'I') spotted in the spectra of the second and third probe is also not identified in the spectrum of first inlet probe. Furthermore, this broadband is also not present in the spectrum of inducer probe and therefore, is expected to be a propagation effect related to either PS cavity and/or inlet duct. The onset of higher order duct modes, specifically the limiting first asymmetric mode at $2.5 \mathrm{kHz}$ for the inlet duct is seen to cause a slight increase in the levels of inlet duct spectra.

Similarly, the analysis of the probes spectra positioned downstream of the impeller (bottom of Fig. 8) shows that he diffuser spectrum has fewer RO tones $\left(1^{\text {st }}\right.$ and $10^{\text {th }} \mathrm{RO}$ and $1^{\text {st }}$ and $\left.2^{\text {nd }} \mathrm{BPF}\right)$ in contrast to the inducer spectrum. This is coherent with the hypothesised mechanism of RO tones [40] as the shock waves corresponding to the sonic conditions at the leading edge of the impeller blades. Therefore, these tones are readily identified at the upstream locations, but not downstream. The low-frequency peak at $0.4 \mathrm{RO}$ is also present in the diffuser spectrum. Furthermore, narrow width broadband noise at $18.8 \mathrm{kHz}$ (highlighted as 'II') corresponding to $11.4 \mathrm{RO}$ is also identified in the diffuser spectrum.

The spectra of the individual sensors forming the outlet piezoelectric sensor array are highly correlated and follow
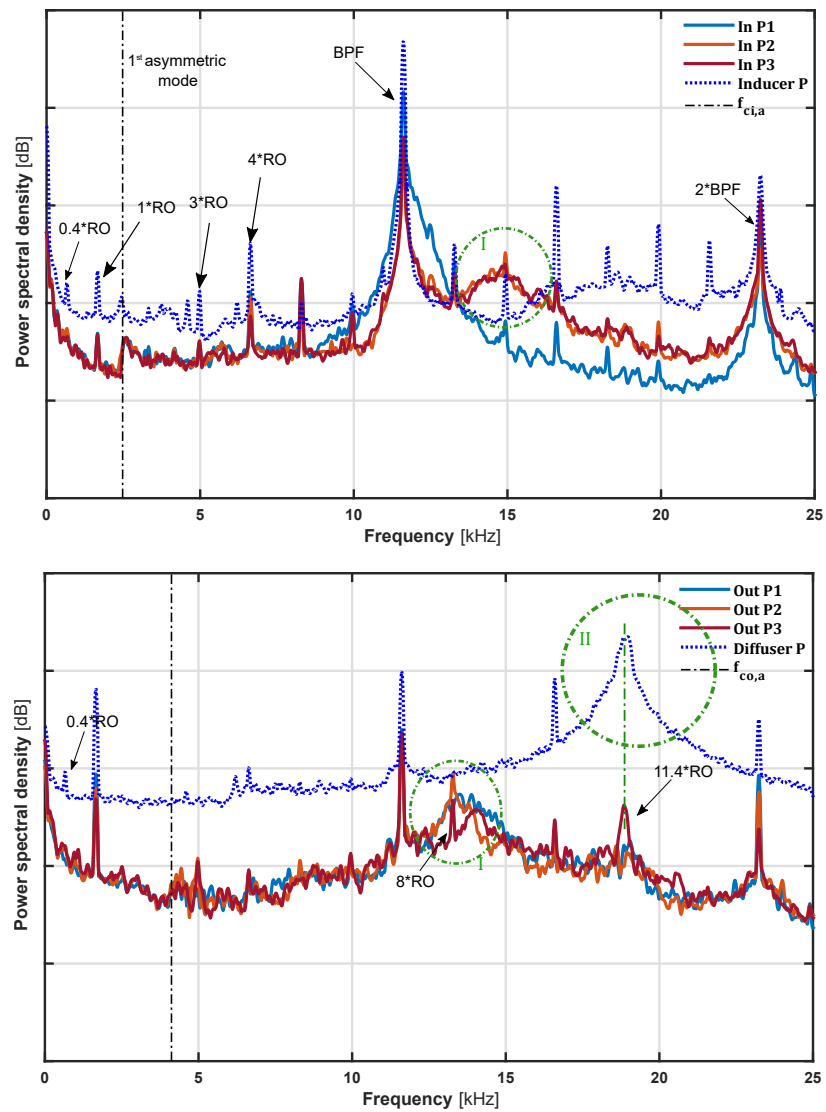

Figure 8: Pressure spectra of the probes located upstream (top) and downstream (bottom) of the impeller for open compressor configuration operating at the design condition of $99 \mathrm{krpm}$ speedline. Former corresponds to inducer and inlet duct array while latter includes diffuser and outlet duct array. Y-axis scale is maintained at $20 \mathrm{~dB}$ per division. 
the diffuser spectra with an anticipated decrease in levels. Again, the broadband feature corresponding to 'whoosh' noise is not identified in the diffuser or outlet duct spectra. Broadband noise in the region of $12.5-14.5 \mathrm{kHz}$ (highlighted as 'I') are identified in the spectra of outlet duct probes but are non existent in the diffuser spectrum, implying a possible propagation feature. Additionally, broadband elevation 'II' seen in the diffuser spectrum does not seem to have propagated to outlet duct. Nevertheless, a peak corresponding to the diffuser broadband 'II' is observed in the spectrum of third outlet probe. Plane wave propagation in the outlet duct is also limited by the onset of the first asymmetric mode at $4.12 \mathrm{kHz}$. The impact of higher order modes is not as significant as seen for inlet duct spectra, although slight variations among otherwise highly coherent outlet spectra can be seen after the cut-off frequency.

An excellent correlation among the spectra of the individual sensors forming inlet and outlet duct arrays points out the precise calibration of individual transducers and complete development of the flow at the measurement sections along with the absence of any reflection component. As for the design operation of the compressor, the flow at the inlet is expected to be steady and uniform while the swirl in the flow exiting the compressor is supposed to settle down by the time it reaches the measurement section. Therefore, the pressure spectrum does not change significantly with the axial position in the array. This being said, deviations are seen in the spectra of the first sensor of the inlet array relative to the other two. This is due to the three-dimensional effects introduced by higher order duct modes as the spectra of three sensors are similar within the plane wave region.

The RO and blade pass harmonics are correlated with the sonic condition at the leading edges, while the broadband elevations marked as 'I' in the spectra of inlet and outlet duct probes are expected to be propagation effects as these broadband noises are not identified in the inducer and diffuser spectra respectively. The broadband elevation marked as 'II' in the diffuser spectra is expected to signify the interaction of the volute tongue with the swirling flow coming out of diffuser.

Near surge operation. In a similar way, the spectra of the compressor operating near surge are analysed. The pressure spectra of the probes located upstream to the impeller (top of Fig. 9) demonstrate the contrast to the design operation with the RO tones non existent in the inducer spectrum of the compressor operating near surge. Nonetheless, BPF and its harmonics can be clearly identified. Broadband elevation in the range of $17-21.5 \mathrm{kHz}$ (marked as 'II') centred around $19 \mathrm{kHz}$ can be seen in the inducer spectrum.

Similar to the design operation, the spectra of the second and third sensor of the inlet duct array are highly correlated while deviation in the spectrum of the first probe can be observed from about $8.2 \mathrm{kHz}$. Furthermore, broadband elevation in the range of $13.5-16 \mathrm{kHz}$ (marked as ' $\mathrm{I}$ ') is identified in the spectra of the second and third sensor of inlet array while not existent in the spectrum of the first sensor. The presence of this broadband ' $\mathrm{I}$ ' in the second and third sensor at both design and near surge operating points further reinforces the hypothesis of it being a propagation effect related to the geometry.

Broadband 'II' identified in the inducer spectrum does not seem to have propagated in the inlet duct. It is interesting to point out the similarity of this broadband with the one seen in the spectrum of the diffuser for the compressor 

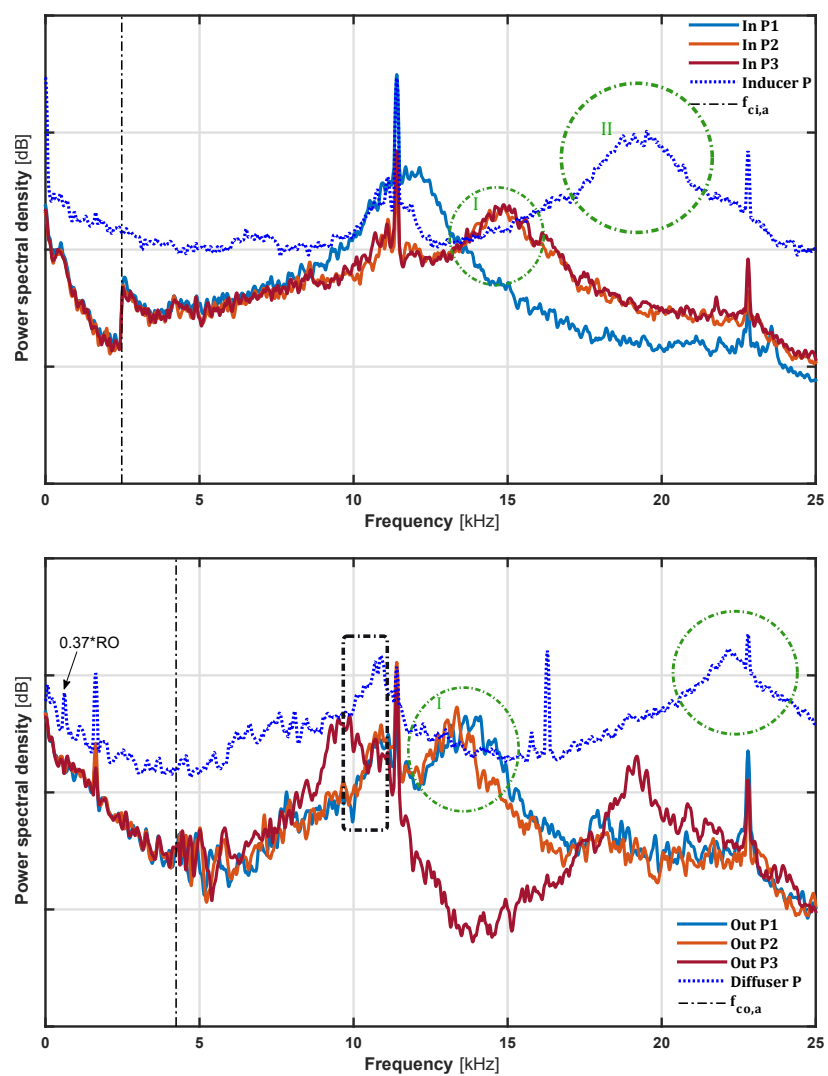

Figure 9: Pressure spectra of the probes located upstream (top) and downstream (bottom) of the impeller for open compressor configuration operating at the near surge condition of $99 \mathrm{krpm}$ speedline. Former corresponds to inducer and inlet duct array while latter includes diffuser and outlet duct array. Y-axis scale is maintained at $20 \mathrm{~dB}$ per division.

operating at design condition (see Fig. 10-bottom plot) for the same iso-speedline. The plane wave propagation in the inlet duct is again limited by the onset of the first asymmetric mode at $2.5 \mathrm{kHz}$ causing a shift in the spectra of inlet duct array. Furthermore, broadband noise corresponding to the 'whoosh' frequency range is not identified in the spectra of the inlet duct for near surge operation either.

The analysis of the spectra of outlet duct array and diffuser probe spectra (bottom of Fig. 9) shows the contrast to the spectrum of the inducer probe with some RO tones $\left(1^{\text {st }}\right.$ and $\left.10^{\text {th }}\right)$ being visible in the diffuser spectrum for near surge operation. In addition to that, a low-frequency tonal noise at approximately $0.37 \mathrm{RO}$ is also identified similar to the $0.4 \mathrm{RO}$ tone in the diffuser spectrum of the design condition. Diffuser spectra levels for the design and near surge operation are reasonably similar as seen the Fig. 10 (middle plot). Broadband elevations in the ranges of 6-11 kHz and 20-22.5 kHz adjacent to the corresponding first and second harmonics of BPF are identified. The former broadband fits into the characteristic Tip Clearance Noise (TCN) frequency range while latter could be either a TCN like feature related to second harmonic of BPF or a broadband feature similar to the one identified in the diffuser spectrum at design conditions but shifted due to the downstream instabilities related to near surge operation. Furthermore, BPF is 


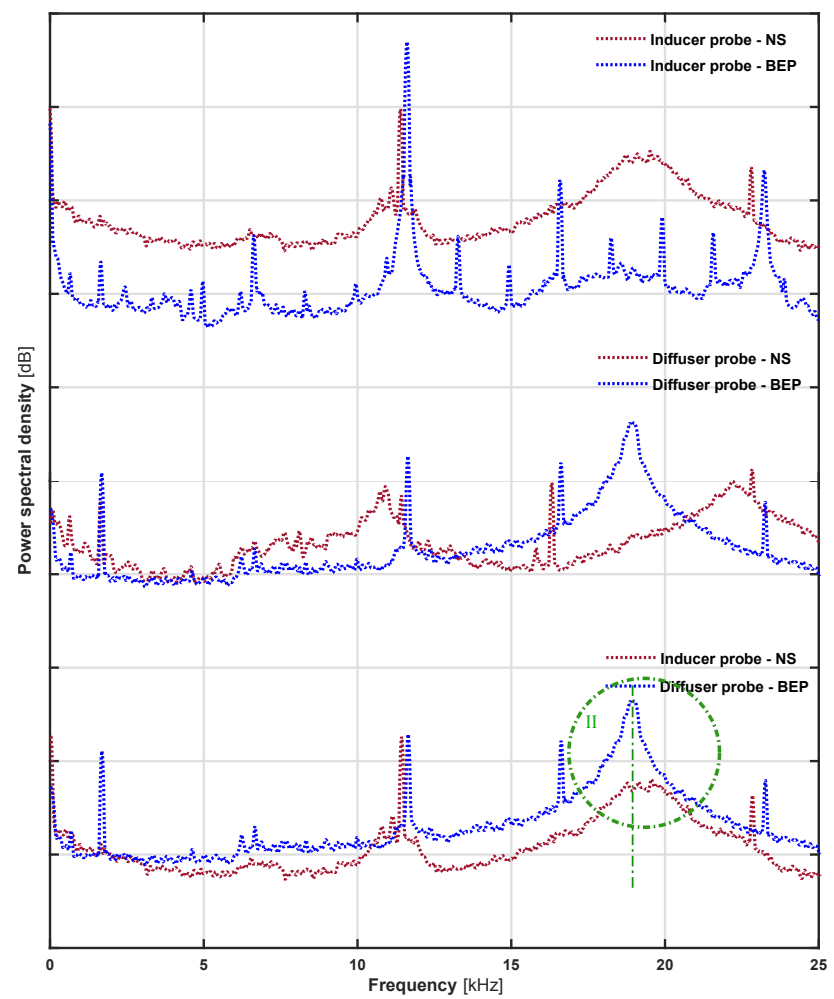

Figure 10: Comparison of the design and near surge pressure spectra for the inducer (top) and diffuser (middle) probes of the open compressor configuration operating at $99 \mathrm{krpm}$. The similarity in the inducer spectrum of near surge operation and diffuser spectrum for design operation is also shown in the bottom plot. Y-axis differences are arbitrary, although the scale is kept at $20 \mathrm{~dB}$ per division.

no longer a distinctive feature as the spectrum is dominated by broadband features.

In contrast to the design condition, the spectra of the first and second sensors of the outlet array are correlated while significant deviation can be seen in the spectrum of the third sensor. Furthermore, broadband elevation in the range of 12.2-15 kHz (marked as 'I') is observed in the spectra of first and second outlet sensors. This is in coherence with the observations made in the outlet spectra at design conditions and reinforces the propagation nature of this broadband feature. The broadband feature similar to the TCN identified in the diffuser spectrum can also be observed in the spectra of the outlet sensors. The broadband adjacent to the second harmonic of BPF does not seem to have propagated to the outlet duct; thereby, making it similar to 'II' broadband instead of TCN. Furthermore, the broadband centred around $19 \mathrm{kHz}$ observed in the diffuser and inducer spectra of design and near surge operation respectively, is also observed in the spectrum of the third sensor of the outlet array. Variations in the spectra of the outlet array's sensor can be seen after the onset of first asymmetric mode. Furthermore, the elevation corresponding to the 'whoosh' noise is not observed either.

The deviations in the spectra of the sensors forming duct arrays can be contributed to the three-dimensional effects caused by duct modes and the propagation of flow instability due to the operation of the compressor near surge. The deviation among the spectra of the inlet duct array is primarily due to duct modes as the flow instability is reported only 
to propagate few diameters upstream [33, 41]. Furthermore, a similar pattern is observed in the inlet spectra at design operation conditions thereby implying this to be a feature dependent on geometry rather than the flow conditions. The deviation in the spectra of the outlet duct array can primarily be attributed to the three-dimensional effects caused by swirl and flow instability present in the compressor outlet. Furthermore, the absence of these deviations in the outlet duct spectra at design conditions further reinforces this reasoning.

The broadband features marked as 'I' in the spectra of inlet and outlet ducts are expected to be caused by either PS geometry or the respective duct modes, as similar features are observed in the corresponding spectra of the design point. The broadband marked as 'II' in the inducer spectrum is hypothesised to be caused by the interaction of the swirling flow in the PS cavity with the structural struts, with three small peaks corresponding to three struts. The mechanism of this broadband is expected to be similar to the one seen in the diffuser spectrum at design conditions, hypothesised to be caused by the interaction of the swirling flow with volute tongue. The resemblance between the two spectra can be seen from the Fig. 10 (bottom plot). The broadband relating to the flow interaction with the volute tongue is not explicitly observed in the same range as seen in the diffuser spectrum of near surge operation. The broadband could have been shifted due to the flow characteristics of near surge operation. Additionally, broadband noise in the characteristic 'whoosh' frequency range is not observed, but a TCN like feature is identified in the diffuser and outlet spectra.

\subsubsection{PS blocked configuration}

The spectra of the PS blocked, and open compressor configurations are compared in this section. The impact of PS casing treatment on the acoustic emission of the compressor is established for both design and near surge operation of the selected iso-speedline.

Design operation. The pressure spectra of the two compressor configurations operating at design conditions are compared in Fig. 11 and Fig. 12. The spectra of the probes located upstream of the impeller are presented in the former figure while latter displays the spectra of the probes positioned downstream of the impeller. The comparison of the spectra of the inlet duct and inducer probes of two configurations (Fig. 11) demonstrates that the inducer spectra for two configurations look similar with the overall levels for the blocked configuration approximately 3-5 dB lower than the open configuration beyond the first harmonic of BPF. In addition to that, a broadband elevation in the range of $6.8-8.8 \mathrm{kHz}$ is identified in the spectrum of the blocked configuration. In contrast to the PS open configuration, the inducer probe is not in direct proximity to the impeller as the PS slot is closed. Therefore, the decreased levels and broadband feature could be the function of the noise propagation in the PS cavity instead of the noise generated by the impeller. Relative reduction in the tonal ( $\mathrm{RO}$ and blade tones) content is observed in the inducer spectrum for the blocked configuration as the rotor-alone pressure does not directly impact the PS cavity. This being said, the low-frequency tone at $0.4 \mathrm{RO}$ is accentuated in the spectrum of the blocked configuration.

The spectra of the probes forming inlet duct array are similar for both configurations in terms of overall levels while the duct spectra of the blocked compressor show a significant reduction in the tonal content. Similar to the 


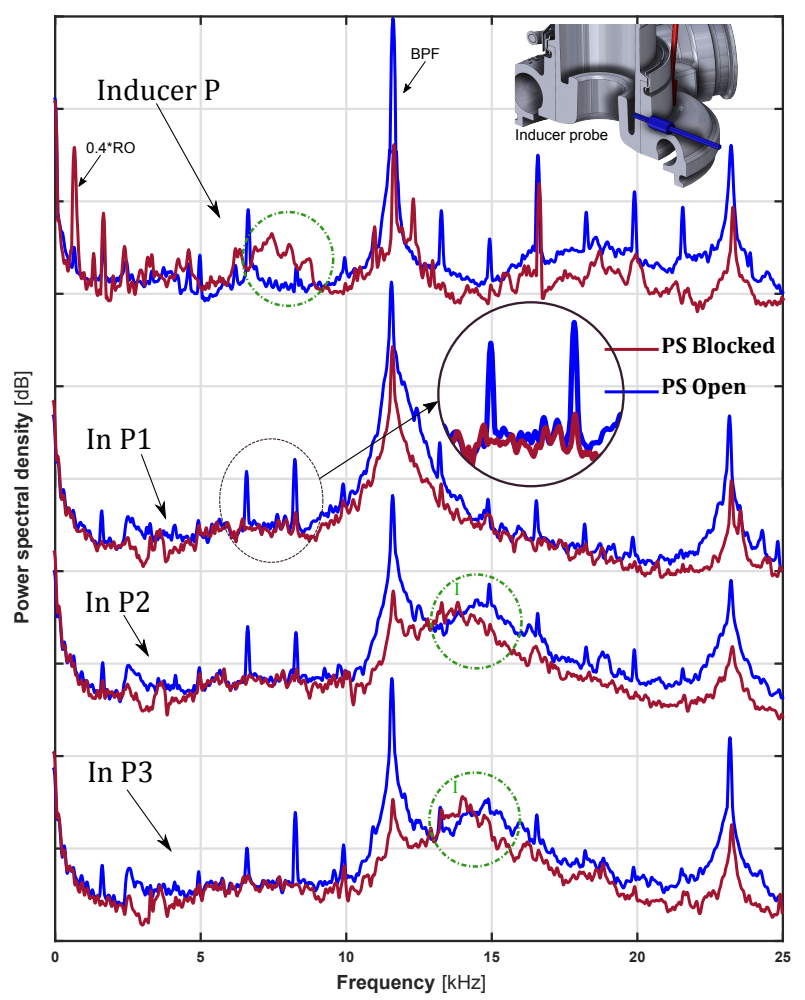

Figure 11: Comparison of the pressure spectra computed from the probes located at the impeller upstream region for the open (blue) and blocked (red) compressors configuration operating at their respective design conditions of $99 \mathrm{krpm}$ speedline. Y-axis differences are arbitrary, although the scale is kept at $20 \mathrm{~dB}$ per division.

spectra of the open configuration, broadband elevation in the range of 13.7-15.7 kHz (highlighted as 'I') can also be observed in the spectra of the second and third inlet probes. This implies that the observed broadband is a propagation characteristic of the inlet duct. The impact of the onset of the first asymmetric mode on inlet duct spectra is again similar to the one observed for the PS open configuration. Furthermore, broadband noise in the 'whoosh' characteristic frequency is not observed either.

The comparison of diffuser and outlet duct probes for the two configurations (Fig. 12) shows that although similar trends are seen in the diffuser spectra of the two configurations, the blocked configuration shows higher overall levels across the frequency range beyond the first rotating tone. Interestingly, the difference in levels is not propagated to the outlet duct as observed from the comparison of duct spectra. Similar to the inducer spectrum, a broadband elevation in the range of 6.2-7.2 kHz is observed in the diffuser spectrum of the PS blocked configuration. Additionally, broadband elevation centred at $18.8 \mathrm{kHz}$ (highlighted as 'II') is identified in the diffuser spectra of both configurations.

Tonal noise corresponding to $1 \mathrm{RO}, \mathrm{BPF}$ and $2 \mathrm{BPF}$ is observed in the diffuser spectra of both configurations while $10 \mathrm{RO}$ is only observed for the open configuration. This implies the possible involvement of PS geometry as the source of 10RO tonal noise. The spectra of the outlet duct probes for two configurations are very similar except for the broadband elevation marked as ' $\mathrm{I}$ ', identified in the spectra of open configuration is not existent in the spectra of 


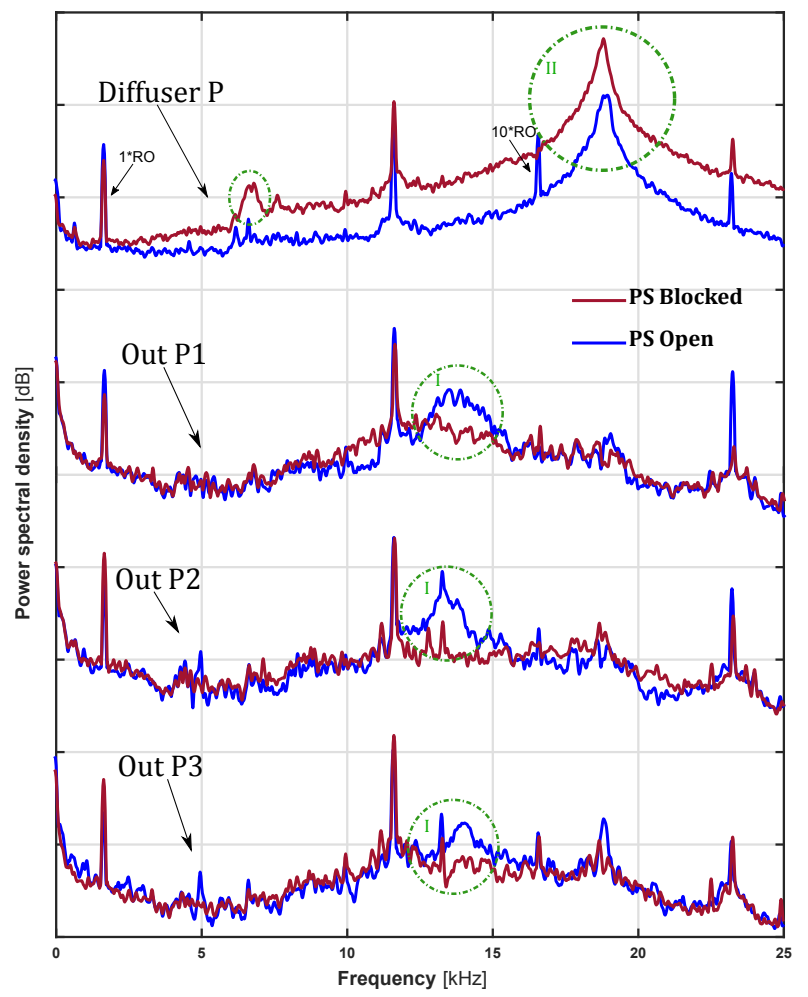

Figure 12: Comparison of the pressure spectra computed from the probes located at the impeller downstream region for the open (blue) and blocked (red) compressors configuration operating at their respective design conditions of $99 \mathrm{krpm}$ speedline. Y-axis differences are arbitrary, although the scale is kept at $20 \mathrm{~dB}$ per division.

blocked configuration. Therefore, this broadband 'I' is expected to be caused by the propagation within the PS cavity. Furthermore, broadband corresponding to the 'whoosh' noise is not observed in the spectra of the outlet duct.

The comparison of the duct spectra of two compressor configurations points that the PS casing treatment does not heavily alter the acoustic emission at the design conditions of the studied iso-speedline. The results do point towards the role of the PS cavity in propagating tonal noise in the direction upstream to the impeller. The comparison of diffuser spectra shows a decrease in the noise generated by flow exiting the impeller by using a PS feature although a tonal noise at 10RO is seen to be caused by the PS. Furthermore, PS casing design is likely causing a broadband noise feature in the outlet duct spectra whilst suppressing a broadband feature in the inducer spectrum.

It is interesting to point out that the RO tones can still be observed in the inducer spectrum of PS blocked configuration operating at the design point which are not observed in the inducer spectrum of a similar compressor but without any casing treatment (Non-PS) investigated by Torregrosa et al. [42]. The general trends of the spectra at the diffuser, inlet and outlet location for PS blocked configuration and Non-PS compressor Torregrosa et al. [42] are reasonably similar. Furthermore, the broadband elevation observed in the diffuser spectra of both PS open and blocked compressor configuration operating at the design condition is also present in the diffuser spectra presented by Torregrosa et al. [42]. 
Near surge operation. The pressure spectra of the probes located at the upstream and downstream of the impeller for two compressor configurations operating near surge are compared in Fig. 13 and Fig. 14 respectively. The comparison of the spectra of the inlet duct and inducer probes of the two configurations (Fig. 13) shows the contrast to the design condition with significant differences in the broadband features seen in the inducer spectra of the two configurations. Broadband elevations corresponding to $2.1-4.3 \mathrm{kHz}$ and $5.5-8.0 \mathrm{kHz}$ observed in the spectrum of the blocked configuration are not existent in the respective spectrum of the open configuration. Another broadband elevation (marked as 'II') centred around $19 \mathrm{kHz}$ is only observed in the spectrum of the open configuration, reinforcing the earlier argument on the nature of this broadband being associated with the flow interaction inside the PS cavity. Furthermore, broadband noise corresponding to TCN is identified in the spectra of both configurations. In addition to the broadband features, peaks at $0.4 \mathrm{RO}$ and $1.5 \mathrm{RO}$ are also observed only in the inducer spectrum of the blocked configuration. Furthermore, significant differences in the overall levels are also observed in the spectra of the two configurations with the blocked compressor registering lower noise levels beyond $9 \mathrm{kHz}$ and relatively higher levels in the frequencies below $9 \mathrm{kHz}$ due to broadband content.

The levels and trends of the inlet duct spectra for the two configurations are similar with the exception of broadband elevations in the range of 3.2-8.5 kHz observed for the blocked configuration. Multiple narrowband elevations in the

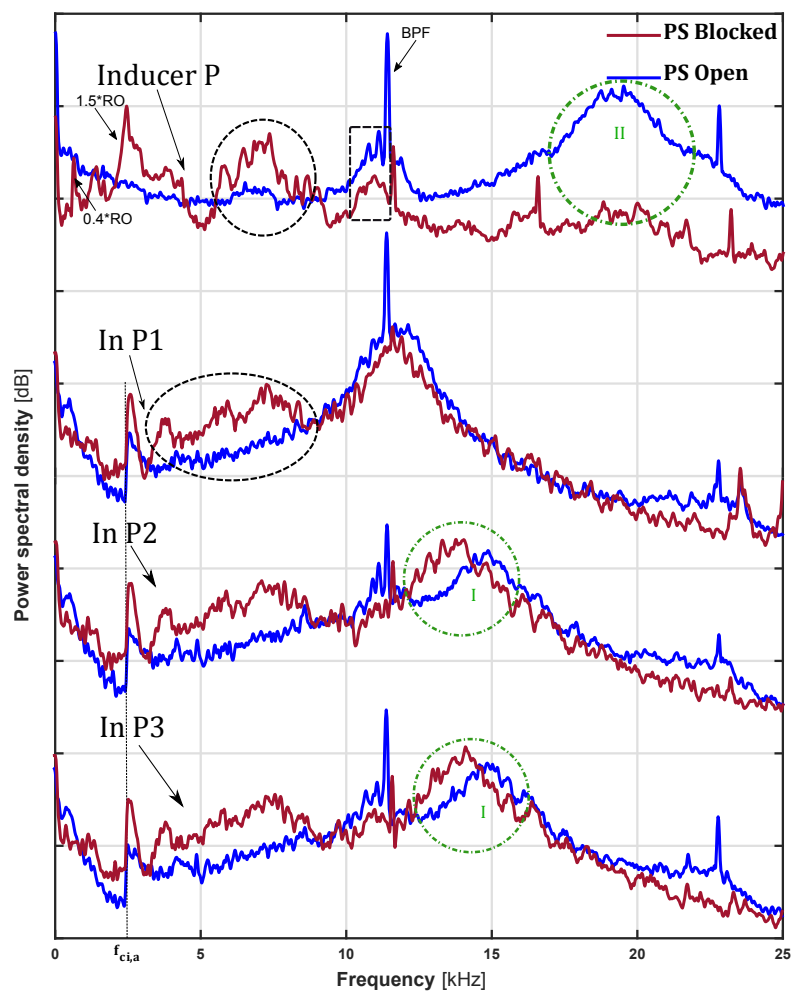

Figure 13: Comparison of the pressure spectra computed from the probes located at the impeller upstream region for the open (blue) and blocked (red) compressors configuration operating at their respective near surge conditions of $99 \mathrm{krpm}$ speedline. Y-axis differences are arbitrary, although the scale is kept at $20 \mathrm{~dB}$ per division. 


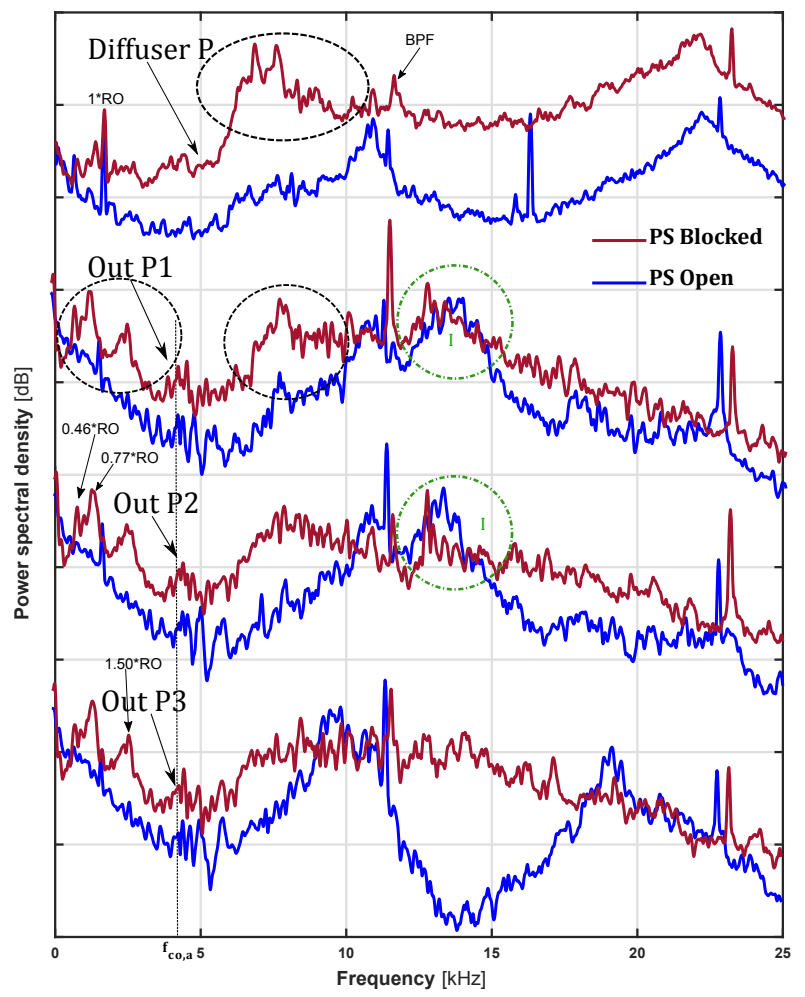

Figure 14: Comparison of the pressure spectra computed from the probes located at the impeller downstream region for the open (blue) and blocked (red) compressors configuration operating at their respective near surge conditions of $99 \mathrm{krpm}$ speedline. Y-axis differences are arbitrary, although the scale is kept at $20 \mathrm{~dB}$ per division.

3.1-4.1 kHz, 5-6.2 kHz and 6.2-8.5 kHz ranges are conflated together as a broadband feature. The latter part of this broadband, i.e. $6.2-8.5 \mathrm{kHz}$ is also observed in the inducer spectrum while the former narrowband, i.e. $3.1-4.1 \mathrm{kHz}$ can be associated with the 'whoosh' noise. The presence of the 3.2-8.5 kHz broadband makes the blocked compressor noisier relative to the open configuration for near surge operation. Broadband highlighted as ' $\mathrm{I}$ ' is again observed in the spectra of both open and blocked configurations implying this is a propagation characteristic of the inlet duct. Furthermore, the tonal noise at $1.5 \mathrm{RO}$ observed in the inducer spectrum of the blocked configuration is also observed in the inlet duct spectra coincident with the onset of first asymmetric mode.

The comparison of the diffuser and outlet duct spectra for two configurations (Fig. 14) demonstrates that the diffuser spectra of the two configurations share a similar trend with higher overall levels for the blocked configuration across the frequency range, specifically beyond the first rotating tone. Similar to the spectra of the upstream probes, a broadband elevation is observed in $5.5-10 \mathrm{kHz}$ is observed in the spectra of the blocked case. Additionally, feeble narrowband elevations in the range of $1.8-3 \mathrm{kHz}$ and $3.2-4.7 \mathrm{kHz}$ are also observed in the blocked spectra with the former corresponding to the 'whoosh' characteristic frequency. Furthermore, BPF is masked by the broadband characteristics in both configurations while tonal noise at $10 \mathrm{RO}$ is not observed in the blocked configuration.

Although the spectra of outlet duct array for the blocked configuration is better correlated than the open config- 
uration, multiple broadband and narrowband features in the ranges of $0.32-1.8 \mathrm{kHz}, 2-3.5 \mathrm{kHz}, 3.8-5 \mathrm{kHz}$ and $6.5-9$ $\mathrm{kHz}$ can be spotted in the outlet duct spectra of the blocked configuration. The first two broadband elevations are in the 'whoosh' noise frequency range, while the last one is consistently seen in the inducer and diffuser spectra. Furthermore, the presence of these broadband features makes the blocked configuration relatively noisier than the corresponding open configuration. Broadband corresponding to TCN which is observed in the spectra of the open configuration is not clearly identifiable in the spectra of the blocked configuration as it seems to be masked by other dominant broadband features. Broadband elevation marked as 'I', identified in the spectra of the open configuration is relatively weaker in the spectra of the blocked configuration, implying its expected propagation within PS cavity.

The comparison of two compressor configurations operating near surge indicates that the PS casing treatment helps in improving the acoustic characteristics of the system. For the investigated iso-speedline, the compressor with the PS design demonstrated lower broadband noise features, including 'whoosh' noise, as well as relatively lower overall noise levels. The noise levels within the plane wave region are relatively similar for the inlet duct while significantly higher levels are seen in the outlet duct of the blocked configuration. The results point out that the alleviation of blockage in the blades passages by the PS design helps with the reduction of broadband noise sources. Also, the PS casing design is likely causing a broadband noise feature (marked as ' $\mathrm{I}$ ') and tonal noise at 10RO in the spectra of the outlet duct and diffuser respectively.

To quantify the differences in the investigated compressor configurations, the overall amplitude of sound pressure level spectra within specific frequency limits are computed from the root mean squared averaged of the pressure signal filtered using bandpass filter for the specified frequency range. Alternatively, the overall levels between frequency bounds $f_{1}$ and $f_{2}$ can also be computed directly from the spectra by

$$
\mathrm{SPL}_{f_{1}-f_{2}}=10 \log _{10}\left(\sum_{i=f_{1}}^{f_{2}} 10^{\mathrm{SPL}_{i} / 10}\right)
$$

The difference in the overall levels between the PS open and the PS blocked compressor configurations within specified frequency limits $(f)$ at particular location $(l)$ i.e. $\Delta_{f, l}=\mathrm{SPL}_{\mathrm{open}}-\mathrm{SPL}_{\mathrm{blocked}}$ for various operating conditions are presented in Tab. 1. The frequency limits includes approximate plane wave regions, BPF and full spectrum while the locations included inducer probe, inlet duct probes, diffuser probe and outlet duct probes. The values for the inlet and outlet duct location are obtained by averaging the individual values of the sensor arrays.

Similarly,the differences in the overall sound intensity levels within plane wave limits for two compressor configurations operating at $99 \mathrm{krpm}$ speedline computed using Eq. 9 are presented in Tab. 2.

$$
\operatorname{SIL}_{f_{1}-f_{2}}=10 \log _{10}\left(10^{12} \sum_{f=f_{1}}^{f_{2}} I(f)\right)
$$

Torregrosa et al. [43] established SIL computed using beamforming as a reliable and robust approach relative to single-sensor SPL approach for plane wave range whereas SPL approach is helpful for investigating the higher 
frequency band. In the current investigation, the differences among the compressor configurations quantified by both SPL and SIL approaches point in a similar general direction. This being said, deviations are observed (see Tab. 1 and Tab. 2) in the amplitude of the differences calculated by two approaches, specifically for the design operation.

Table 1: Differences in the overall sound pressure levels of PS open and blocked compressor configuration.

\begin{tabular}{|c|c|c|c|c|c|}
\hline \multicolumn{6}{|c|}{$\Delta_{f, l}=\mathrm{SPL}_{\text {open }}-\mathrm{SPL}_{\text {blocked }}[\mathrm{dB}]$} \\
\hline \multirow{2}{*}{ Operating point } & \multirow{2}{*}{ Frequency range $[\mathrm{kHz}]$} & \multicolumn{4}{|c|}{ Location } \\
\hline & & $\Delta$ Inducer & $\Delta$ Inlet & $\Delta$ Diffuser & $\Delta$ Outlet \\
\hline \multirow{3}{*}{99 krpm - Design } & Plane wave limit ${ }^{3}$ & -1.7 & 6.4 & 1.6 & -1.8 \\
\hline & $\mathrm{BPF}(0.2-11.6)$ & 5.4 & 12.1 & -4.2 & -1.8 \\
\hline & $0.02-25$ & 22.7 & 12.4 & -16.3 & -2.0 \\
\hline \multirow{3}{*}{99 krpm - NS } & Plane wave limit & -25.9 & 1.2 & -20.3 & -5.3 \\
\hline & $\mathrm{BPF}(0.2-11.6)$ & -5.5 & -6.9 & -13.2 & -3.3 \\
\hline & $0.02-25$ & 6.5 & -8.3 & -13.8 & -18.2 \\
\hline \multirow{3}{*}{130 krpm - Design } & Plane wave limit ${ }^{4}$ & 5.1 & 1.1 & 0.4 & 0.7 \\
\hline & BPF $(0.2-15.33)$ & 3.9 & 9.6 & 0.3 & 11.6 \\
\hline & $0.02-35$ & -0.3 & 10.5 & -0.7 & 12.0 \\
\hline
\end{tabular}

Table 2: Differences in the overall sound intensity levels of PS open and blocked compressor configuration operating $99 \mathrm{krpm}$ speedline

\begin{tabular}{ccccc}
\multicolumn{5}{c}{$\Delta_{f, l}=\mathrm{SIL}_{\text {open }}-\mathrm{SIL}_{\text {blocked }}[\mathrm{dB}]$} \\
\hline \multirow{2}{*}{ Speedline } & Operating point & Frequency range $[\mathrm{kHz}]$ & $\Delta$ Inlet & $\Delta$ Outlet \\
\hline \multirow{2}{*}{$99 \mathrm{krpm}$} & BEP & Plane wave limit ${ }^{3}$ & 0.4 & -1.9 \\
& $\mathrm{NS}$ & -1.1 & -4.7 \\
\hline
\end{tabular}

\section{2. $130 \mathrm{krpm}$ speedline}

Acoustic characterisation of the open and blocked compressor configurations operating at the $130 \mathrm{krpm}$ speedline is intended to serve two objectives. Firstly, comparing the acoustic spectra of the $130 \mathrm{krpm}$ with the $99 \mathrm{krpm}$ speed line would help in understanding the evolution of the acoustic behaviour with an increase in operational speed. Secondly,

\footnotetext{
${ }^{3}$ Frequency bounds of the plane wave propagation for $99 \mathrm{krpm}$ speedline are approximated to be $0.02-2.5 \mathrm{kHz}$ and $0.02-4.0 \mathrm{kHz}$ for inlet and outlet duct respectively.

${ }^{4}$ Frequency bounds of the plane wave propagation for $130 \mathrm{krpm}$ speedline are approximated to be $0.02-2.5 \mathrm{kHz}$ and $0.02-4.5 \mathrm{kHz}$ for inlet and outlet duct respectively.
} 
the impact of the PS design at higher speeds is explored by comparing the spectra of open and blocked compressor configurations operating at $130 \mathrm{krpm}$. It must be noted that the design states of two speedlines for both configurations are aerodynamically similar. Therefore, the inferences on the impact of operational speed and PS cavity can only be made by comparing the spectra of design points for $99 \mathrm{krpm}$ with the $130 \mathrm{krpm}$ speedline, and open and blocked configurations operating at $130 \mathrm{krpm}$ respectively. The deviation in the near surge spectra of the two speedlines for both configurations are not only dependent on operating speed but on the different aerodynamic conditions too. Therefore, the direct comparison between the near surge states of the $99 \mathrm{krpm}$ and $130 \mathrm{krpm}$ speedlines is not correct. In this work, the near surge spectra are only used to draw a broader understanding instead of quantifying the impact of operation speed.

\subsubsection{PS open configuration}

The spectra of the various probes for the compressor operating at the design and near surge conditions of 130 krpm iso-speedline are presented in Fig. 15 and Fig. 16 respectively. The spectra of two iso-speedlines are similar in trend, with the higher speedline showing an increase of 5-10 dB in the overall levels. Furthermore, the noise features,
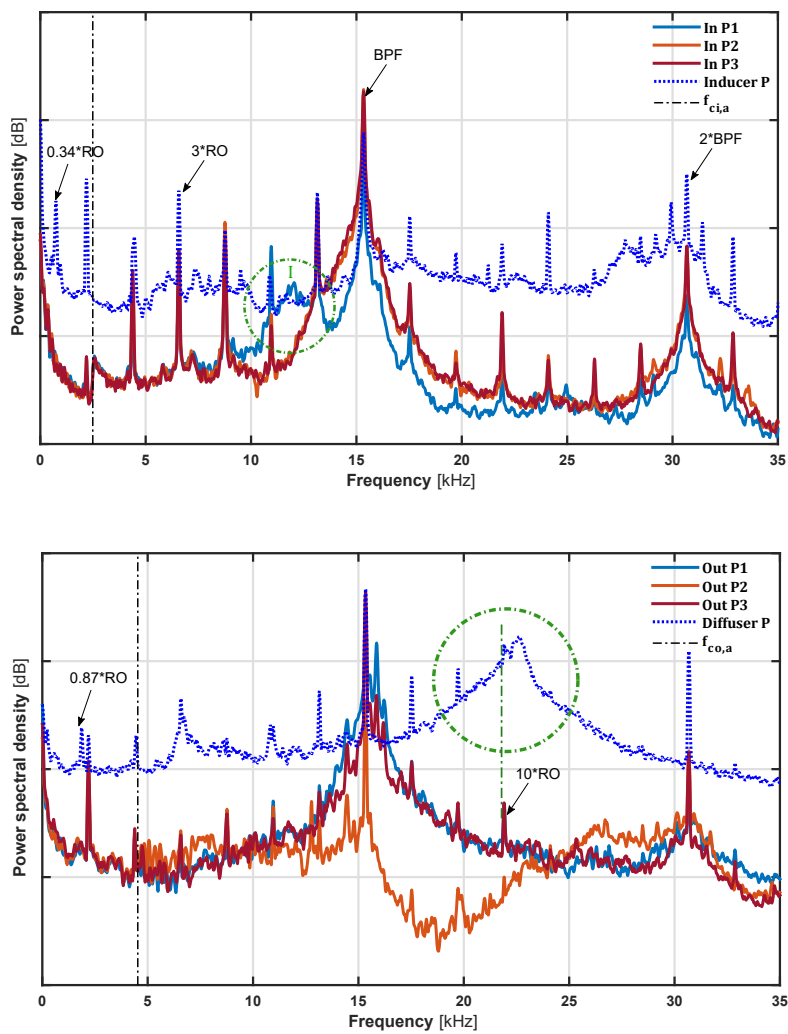

Figure 15: Pressure spectra of the probes located upstream (top) and downstream (bottom) of the impeller for open compressor configuration operating at the design condition of $130 \mathrm{krpm}$ speedline. Former corresponds to inducer and inlet duct array while latter includes diffuser and outlet duct array. Y-axis scale is maintained at $20 \mathrm{~dB}$ per division. 
both tonal and broadband are seen to have shifted commensurately with the change in operation speed.

The impact of operational speed on acoustic emission for design operation inferred from comparing the spectra of $99 \mathrm{krpm}$ (Fig. 8), and $130 \mathrm{krpm}$ iso-speedlines (Fig. 15) shows that the RO or 'buzz-saw' tones are accentuated in the inducer spectrum with an increase in rotational speed. Furthermore, the low-frequency tonal noise observed at $0.66 \mathrm{kHz}(0.4 \mathrm{RO})$ in the spectrum of $99 \mathrm{krpm}$ speedline is seen to have slightly shifted to $0.74 \mathrm{kHz}(0.34 \mathrm{RO})$ in the spectrum $130 \mathrm{krpm}$ speedline. The spectra of the piezoelectric sensor forming inlet duct array showcase similar trends as seen in the lower speedline, with the spectra of second and third sensors strongly correlated while some deviations can be observed in the spectra of the first sensor. Furthermore, a broadband elevation at 13.7-15.7 kHz (marked as 'I') observed in the spectra of the second and third inlet sensor of lower speedline seems to have shifted in the range of $10-14 \mathrm{kHz}$ for $130 \mathrm{krpm}$ speedline and is only observed in the spectra of first inlet sensor.

The spectrum of the diffuser probe also shows higher tonal content with the increase in rotational speed. In addition to that, the lower frequency tonal peak has shifted from $0.66 \mathrm{kHz}$ to $1.87 \mathrm{kHz}$ with an increase in speed. Furthermore, a similar trend is seen with the shift in the broadband elevation centred on $18.8 \mathrm{kHz}$ for the lower speedline to $22.6 \mathrm{kHz}$ for the higher speedline. The spectra of the outlet duct array for the higher speed line show deviations in correlation as
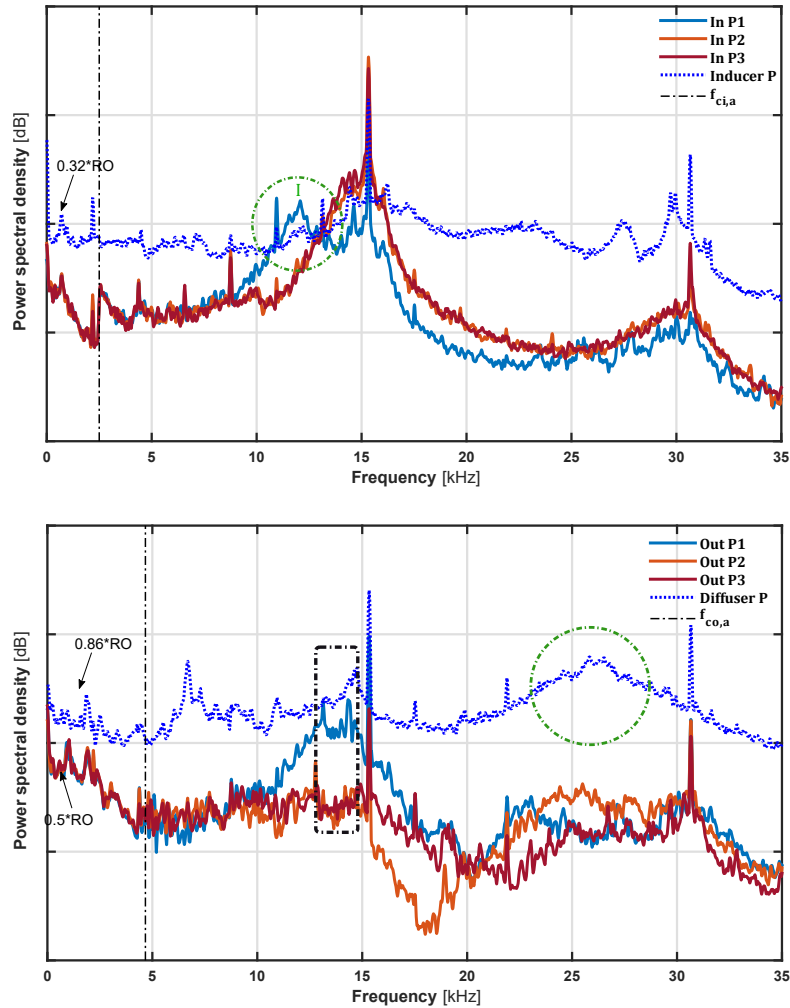

Figure 16: Pressure spectra of the probes located upstream (top) and downstream (bottom) of the impeller for open compressor configuration operating at the near surge condition of $130 \mathrm{krpm}$ speedline. Former corresponds to inducer and inlet duct array while latter includes diffuser and outlet duct array. Y-axis scale is maintained at $20 \mathrm{~dB}$ per division. 
compared to the lower speed line. Also, broadband elevation seen in the range of $12.5-14.5 \mathrm{kHz}$ in the outlet spectra of the lower speed line is not existent in the corresponding spectra of higher speedline. Analysis of the compressor configurations at the lower speedline pointed to the role of the PS cavity in the presence of this broadband and possible cause for its non-existence is discussed in the later part of this section.

As mentioned before, the direct comparison of the spectra of near surge points does not present an accurate impact of the rotational speed on the emission spectra for near surge operation. Nevertheless, the comparison of the near surge spectra of $130 \mathrm{krpm}$ (Fig. 16) and $99 \mathrm{krpm}$ (Fig. 9) speedlines demonstrates that although an increase in the tonal content is observed in the inducer spectrum of higher speed line, the broadband (marked as 'II') in the inducer spectrum of the lower speedline is shifted and observed faintly for the higher speedline. As this broadband was expected to be associated with the interaction of the flow in PS cavity, the reasons for its fading is discussed later in this section.

Similar to the design point, the broadband marked as 'I' in the inlet duct spectra of the $99 \mathrm{krpm}$ speedline is shifted in the inlet duct spectra of the higher speed line. In addition to that, the shifts in the tonal and broadband noise features are seen with the increase in the rotational speed. Furthermore, broadband marked as 'I' in the outlet duct spectra of

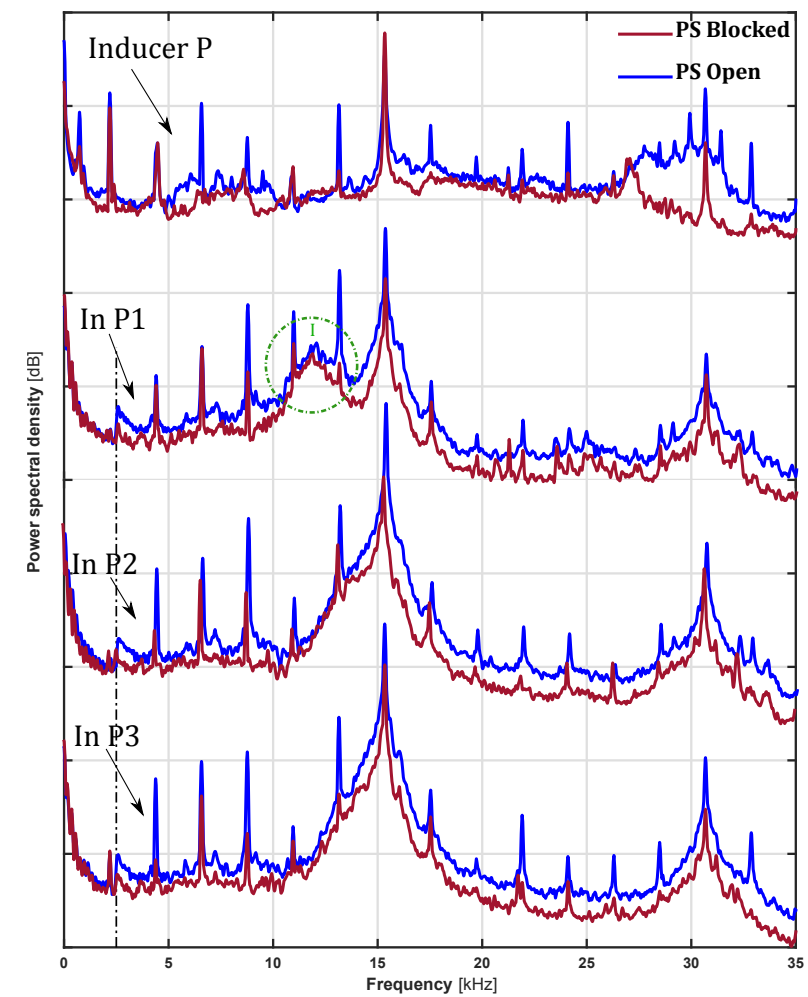

Figure 17: Comparison of the pressure spectra computed from the probes located at the impeller upstream region for the open (blue) and blocked (red) compressors configuration operating at their respective design conditions of $130 \mathrm{krpm}$ speedline. Y-axis differences are arbitrary, although the scale is kept at $20 \mathrm{~dB}$ per division. 
the lower speed line is also not observed in the corresponding spectra of the higher speed line.

The results clearly show an increase in overall noise levels and tonal content with an increase in the rotational speed for the operation of the compressor at design conditions. The increase in tonal noise is expected, as the higher tip speed would lead to severe transonic conditions and shock waves attached to the blade in the rotor alone pressure field. The shifts and decrease in the strength of the features associated with the recirculating flow in the PS cavity are expected due to the decreased flow recirculation in the PS cavity at higher operating speeds as shown by Sharma et al. [33].

\subsubsection{PS blocked configuration}

The spectra of the PS blocked, and open compressor configurations operating at design conditions are compared in this section to understand the impact of PS casing treatment at higher operating speed. The spectra of the probes located upstream of the impeller are compared in the Fig. 17 while Fig. 18 shows the spectra of the probes positioned downstream of the impeller.

In contrast to the lower speedline ( $99 \mathrm{krpm})$, the differences in the noise generated by the compressors (inducer and diffuser spectra) for two configurations are insignificant. The expected reduction of the propagation of the tonal

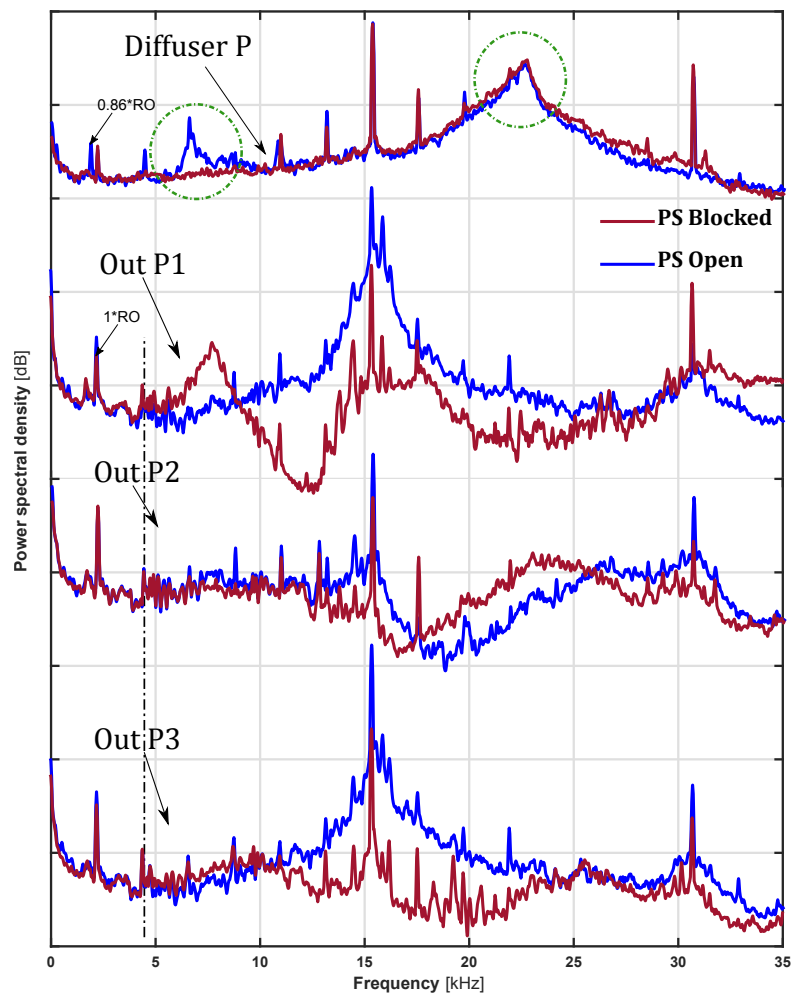

Figure 18: Comparison of the pressure spectra computed from the probes located at the impeller downstream region for the open (blue) and blocked (red) compressors configuration operating at their respective design conditions of $130 \mathrm{krpm}$ speedline. Y-axis differences are arbitrary, although the scale is kept at $20 \mathrm{~dB}$ per division. 
noise in the upstream impeller direction for the blocked configuration can be observed in the spectra of inducer and inlet duct probes. This reconfirms the role of the PS design in the upstream propagation of RO or 'buzz-saw' tones [40] for the design operation of the compressor. The spectra of the probes positioned downstream of the impeller also shows similar results with insignificant impact on the overall levels, specifically within the plane wave region. Broadband elevation in the range of $6-8 \mathrm{kHz}$ observed in the diffuser spectrum of the open configuration is not present in the corresponding blocked spectra. The outlet duct spectra indicate a quieter operation of the blocked configuration in the mid-frequency range of $10-20 \mathrm{kHz}$. While the impact of PS is limited to tonal propagation for the design condition, significant broadband impact is expected for near surge operating conditions. Again, the decreased impact of PS at higher operating speed can be attributed to the reduced flow recirculation via PS cavity.

\section{Conclusions}

An experimental campaign to characterise the acoustic behaviour of the compressor is presented in this work. The impact of PS casing treatment on the acoustics of the compressor was evaluated using open and blocked compressor configurations. Aerodynamically similar design and near surge operating points on the $99 \mathrm{krpm}$ speedline for the two compressor configurations were selected for acoustic measurements. Additionally, the impact of operational speed on the acoustics was quantified by comparing aerodynamically similar operating points across $99 \mathrm{krpm}$ and $130 \mathrm{krpm}$ speedlines.

An in-duct noise measurement method was used to characterise the acoustic emission of the compressor. The noise generated in the compressor was quantified by measuring pressure fluctuations near the inducer and diffuser while propagation of the generated noise was computed from an array of piezoelectric sensors in the inlet and outlet ducts.

The inducer spectra for the design conditions are dominated by tonal noise which includes RO or 'buzz-saw' tones and harmonics of BPF. The upstream propagation of tonal noise in the open compressor configuration can be identified in the spectra of the inlet duct while downstream propagation of these tones is limited, with only a few RO tones and BPF being observed in the diffuser spectrum. Although flat, an interesting broadband feature (marked as 'II') is identified in the diffuser spectrum. This is thought to be caused by the interaction of the swirling flow with the volute tongue. The spectra of the inlet and outlet duct arrays follow the general trends observed in the corresponding inducer and diffuser spectra with a few deviations caused by propagation effects. The broadband feature 'II' observed in the diffuser spectrum is not seen to be propagated to the outlet duct. Additionally, broadband features highlighted as 'I' are also observed in the inlet and outlet duct spectra. The broadband feature observed in the inlet duct is thought to be caused by three-dimensional effects judged to be caused by the propagation effects introduced by the PS cavity.

In contrast to the design conditions, a lack of RO tones is observed in the spectra of the inducer for near surge conditions. The spectra are dominated by broadband content with only BPF as an observed tonal feature. A broadband feature similar to the broadband 'II' seen in the diffuser spectra of the design point is observed in the spectrum of 
the inducer probe for the open compressor configuration operating near surge. This is thought to be caused by the interaction of swirling flow in the PS cavity with the structural struts. The first RO tone can be observed in the spectra of the diffuser point, but the BPF tone is feeble. Similar to the design spectra, broadband features 'I' are also observed in the inlet and outlet duct spectra for near surge point. Furthermore, broadband corresponding to TCN can also be observed in the diffuser and outlet duct spectra. Characteristic 'whoosh' noise broadband elevation is not observed for the open compressor configuration operating at $99 \mathrm{krpm}$.

For the design operation, whilst the PS cavity does not significantly alter the overall noise levels of the compressor, an increase of around 3-5 dB is seen in the frequency region beyond $10 \mathrm{kHz}$ for the $99 \mathrm{krpm}$ speedline. The casing treatment does seem to propagate tonal noise in the direction upstream of the impeller as seen from the comparison of inlet duct spectra, while the spectra of the outlet duct remain largely unchanged. On the other hand, acoustic characteristics of the compressor operating near surge are positively impacted by the PS casing treatment, causing a reduction of approximately $10-15 \mathrm{~dB}$ in the range up to blade pass frequency. Various broadband features in the frequency ranges of 'whoosh' and TCN that are observed in the inlet and outlet duct spectra of the blocked configuration are alleviated by the PS casing treatment. It is interesting to point out that the comparison of inducer and diffuser spectra of the two configurations implies that the noise generated in the impeller upstream region increases while in the impeller downstream region it decreases by the use of PS casing treatment. The higher levels of noise at the inducer probe for the open configuration could be due to the direct proximity to the rotating impeller which is not available for the blocked configuration.

An increase in operational speed shows an expected increase in the noise levels, specifically accentuated RO tonal features seen for the design operation. An expected shift in the frequency range of various spectral features due to changes in the rotational speed can also be observed in the spectra of the higher speedline. It is interesting to point out that the broadband feature ' $\mathrm{I}$ ' observed in the outlet duct for the operating points on the $99 \mathrm{krpm}$ line is not observed for corresponding points on the $130 \mathrm{krpm}$ speedline. Furthermore, broadband 'II' observed in the inducer spectrum of the near surge for $99 \mathrm{krpm}$ is weak in the corresponding spectrum of the $130 \mathrm{krpm}$ speedline. Both of these broadband features are associated with the PS cavity, and their strength is observed to decrease as the flow recirculated via PS cavity reduces at higher operating speed.

The impact of PS casing treatment on the compressor operating at the design condition on the higher speedline is similar to the lower speedline but with reduced overall deviation. The noise generated for the two configurations at $130 \mathrm{krpm}$ is similar, inferring reduced flow dynamics within the PS cavity at higher speedlines.

\section{Acknowledgements}

The project was sponsored and supported by BorgWarner Turbo Systems and the Regional Growth Fund (RGF Grant Award 01.09.07.01/1789C). The authors would like to thank BorgWarner Turbo Systems for permission to publish the results presented in this paper. 


\section{References}

[1] X. He, X. Zheng, Roles and mechanisms of casing treatment on different scales of flow instability in high pressure ratio centrifugal compressors, Aerospace Science and Technology 84 (2019) 734-746. doi:10.1016/j.ast.2018.10.015.

[2] S.-B. Ma, K.-Y. Kim, Optimization of discrete cavities in a centrifugal compressor to enhance operating stability, Aerospace Science and Technology 68 (2017) 308-319. doi:10.1016/j.ast.2017.05.029.

[3] C. Yang, W. Wang, H. Zhang, C. Yang, Y. Li, Investigation of stall process flow field in transonic centrifugal compressor with volute, Aerospace Science and Technology 81 (2018) 53-64. doi:10.1016/j.ast.2018.07.047.

[4] D. Evans, A. Ward, Minimising Turbocharger Whoosh Noise for Diesel Powertrains, SAE Paper : SAE Noise and Vibration Conference and Exhibition, Society of Automotive Engineers, Warrendale, Penn., 2005. doi :10.4271/2005-01-2485.

[5] H. Sun, S. Lee, Numerical prediction of centrifugal compressor noise, Journal of sound and vibration 269 (1) (2004) 421-430. doi: 10.1016/S0022-460X (03) 00330-4.

[6] H. Sun, H. Shin, S. Lee, Analysis and optimization of aerodynamic noise in a centrifugal compressor, Journal of Sound and Vibration 289 (4-5) (2006) 999-1018. doi:10.1016/j.jsv.2005.03.004.

[7] C. Teng, S. Homco, Investigation of compressor whoosh noise in automotive turbochargers, SAE Int. J. Passeng. Cars - Mech. Syst. 2 (1) (2009) 1345-1351. doi:10.4271/2009-01-2053.

[8] Z. Sun, W. Zou, X. Zheng, Instability detection of centrifugal compressors by means of acoustic measurements, Aerospace Science and Technology 82 (2018) 628-635. doi:10.1016/j.ast.2018.09.006.

[9] Y. W. Lee, D. J. Lee, Y. So, D. Chung, Control of airflow noise from diesel engine turbocharger, Tech. rep., SAE Technical Paper (2011). doi:10.4271/2011-01-0933.

[10] A. V. Pai, S. Walsh, D. O’Boy, R. Chen, Turbocharger surge noise measurement and solution using experimental techniques, in: 22nd International Congress on Sound and Vibration, Vol. ICSV22, 2015.

[11] A. V. Pai, S. J. Walsh, D. J. O’Boy, R. Chen, Air intake system noise in a turbocharged petrol engine during transient operation, in: 42nd International Congress and Exposition on Noise Control Engineering 2013, INTER-NOISE 2013: Noise Control for Quality of Life, Vol. 1, pp. 288-295.

[12] G. Gaude, T. Lefevre, R. Tanna, K. Jin, T. J. B. McKitterick, S. Armenio, Experimental and computational challenges in the quantification of turbocharger vibro-acoustic sources, INTER-NOISE and NOISE-CON Congress and Conference Proceedings 2008 (3) (2008) $5754-5767$.

[13] H. Tiikoja, H. Rämmal, M. Abom, H. Boden, Investigations of automotive turbocharger acoustics, SAE International Journal of Engines 4 (2) (2011) 2531-2542. doi : 10.4271/2011-24-0221.

[14] T. Raitor, W. Neise, Sound generation in centrifugal compressors, Journal of sound and vibration 314 (3) (2008) 738-756. doi:10.1016/ j.jsv.2008.01.034.

[15] A. Broatch, J. Galindo, R. Navarro, J. García-Tíscar, Methodology for experimental validation of a cfd model for predicting noise generation in centrifugal compressors, The International journal of heat and fluid flow 50 (2014) 134-144. doi:10.1016/j.ijheatfluidflow. 2014.06 .006

[16] A. J. Torregrosa, A. Broatch, R. Navarro, J. García-Tíscar, Acoustic characterization of automotive turbocompressors, International Journal of Engine Research 16 (1) (2015) 31-37. doi : 10.1177/1468087414562866.

[17] E. P. Trochon, A new type of silencers for turbocharger noise control, Tech. rep., SAE Technical Paper (2001). doi:10.4271/ 2001-01-1436.

[18] A. Broatch, J. García-Tíscar, F. Roig, S. Sharma, Dynamic mode decomposition of the acoustic field in radial compressors, Aerospace Science and Technologydoi:10.1016/j.ast.2019.05.015.

[19] A. J. Torregrosa, A. Broatch, X. Margot, J. García-Tíscar, Experimental methodology for turbocompressor in-duct noise evaluation based on beamforming wave decomposition, Journal of Sound and Vibration 376 (2016) 60-71. doi:10.1016/j.jsv.2016.04.035.

[20] C. Sevginer, M. O. Arslan, N. Sonmez, S. I. Yilmaz, Investigation of turbocharger related whoosh and air blow nosie in a diesel powertrain, in: INTER-NOISE and NOISE-CON Congress and Conference Proceedings, Vol. 2007, Institute of Noise Control Engineering, pp. $3163-3172$. 
[21] N. Figurella, R. Dehner, A. Selamet, K. Tallio, K. Miazgowicz, R. Wade, Noise at the mid to high flow range of a turbocharger compressor, Noise Control Engineering Journal 62 (5) (2014) 306-312. doi : 10.3397/1/376229.

[22] N. Figurella, R. Dehmer, A. Selamet, K. Tallio, K. Miazgowicz, R. Wade, A. Karim, P. Keller, J. Shutty, Effect of inlet vanes on centrifugal compressor acoustics and performance, Noise Control Engineering Journal 62 (4) (2014) 232-237. doi : 10. $3397 / 1 / 376223$.

[23] H. Chen, J. Yin, Turbocharger compressor development for diesel passenger car applications A2 - Group, Institution of Mechanical Engineers Combustion Engines \& Fuels, Woodhead Publishing, 2006, pp. 15-27. doi : 10.1016/B978-1-84569-174-5.50005-1.

[24] H. Chen, V.-M. Lei, Casing treatment and inlet swirl of centrifugal compressors, Journal of Turbomachinery 135 (4) (2013) 041010.

[25] R. Dehner, A. Selamet, M. Steiger, K. Miazgowicz, A. Karim, The effect of ported shroud recirculating casing treatment on turbocharger centrifugal compressor acoustics 10 (2017) 2057-2066. doi :10.4271/2017-01-1796.

[26] N. Fardafshar, P. Koutsovasilis, Ported shroud influence on the aero-acoustic properties of automotive turbochargers: Quantification by means of simulation and measurement, 2018.

[27] C. L. Morfey, Sound transmission and generation in ducts with flow, Journal of Sound and Vibration 14 (1) (1971) 37-55. doi:10.1016/ 0022-460X (71) 90506-2.

[28] J. García Tíscar, Experiments on turbocharger compressor acoustics, Thesis doctoral (2017). doi : 10.4995/Thesis/10251/79552.

[29] A. J. Torregrosa, A. Broatch, V. Bermúdez, I. Andrés, Experimental assessment of emission models used for ic engine exhaust noise prediction, Experimental Thermal and Fluid Science 30 (2) (2005) 97-107. doi:10.1016/j.expthermflusci.2005.05.001.

[30] L. J. Eriksson, Higher order mode effects in circular ducts and expansion chambers, The Journal of the Acoustical Society of America 68 (2) (1980) 545-550. doi:10.1121/1.384768.

[31] M. Åbom, H. Bodén, Error analysis of two-microphone measurements in ducts with flow, The Journal of the Acoustical Society of America 83 (6) (1988) 2429-2438. doi:10.1121/1.396322.

[32] B. T. Systems, Bradford, uk. URL http: //turbos. borgwarner.com

[33] S. Sharma, M. L. Jupp, A. K. Nickson, J. M. Allport, Ported shroud flow processes and their effect on turbocharger compressor operation, in: ASME Turbo Expo 2017: Turbomachinery Technical Conference and Exposition, American Society of Mechanical Engineers, 2017, pp. V02CT44A017-V02CT44A017. doi:10.1115/GT2017-63678.

[34] E. M. Greitzer, The stability of pumping systems - the 1980 freeman scholar lecture, Journal of Fluids Engineering 103 (2) (1981) $193-242$. doi: $10.1115 / 1.3241725$.

[35] J. Weidemann, Analysis of the relations between acoustic and aerodynamic parameters for a series of dimensionally similar centrifugal fan rotors, NASA Tech. Trans. 13 (1971) 1.

[36] W. Neise, Application of similarity laws to the blade passage sound of centrifugal fans, Journal of Sound and Vibration 43 (1) (1975) 61-75. doi:10.1016/0022-460x (75)90205-9.

[37] W. Neise, B. Barsikow, Acoustic similarity laws for fans, Journal of Engineering for Industry 104 (2) (1982) $162-168$. doi:10.1115/1. 3185811.

[38] W.-H. Jeon, D.-J. Lee, H. Rhee, An application of the acoustic similarity law to the numerical analysis of centrifugal fan noise, JSME international journal. Series C, Mechanical systems, machine elements and manufacturing 47 (3) (2004) 845-851. doi:10.1299/jsmec . 47.845 .

[39] P. Welch, The use of fast fourier transform for the estimation of power spectra: A method based on time averaging over short, modified periodograms, IEEE Transactions on Audio and Electroacoustics 15 (2) (1967) 70-73. doi : 10.1109/TAU.1967.1161901.

[40] S. Sharma, A. Broatch, J. Garcia-Tiscar, J. M. Allport, A. K. Nickson, Acoustic characteristics of a ported shroud turbocompressor operating at design conditions, International J of Engine Research 1 (2018) 15. doi : 10.1177/1468087418814635.

[41] G. Després, G. N. Boum, F. Leboeuf, D. Chalet, P. Chesse, A. Lefebvre, Simulation of near surge instabilities onset in a turbocharger compressor, Proceedings of the Institution of Mechanical Engineers, Part A: Journal of Power and Energy 227 (6) (2013) 665-673. doi : $10.1177 / 0957650913495537$. 
[42] A. Torregrosa, A. Broatch, X. Margot, J. García-Tíscar, Y. Narvekar, R. Cheung, Local flow measurements in a turbocharger compressor inlet, Experimental Thermal and Fluid Science 88 (2017) 542-553. doi:10.1016/j .expthermflusci.2017.07.007.

[43] A. J. Torregrosa, A. Broatch, R. Navarro, J. García-Tíscar, Acoustic characterization of automotive turbocompressors, International Journal of Engine Research 16 (1) (2015) 31-37. doi:10.1177/1468087414562866. 